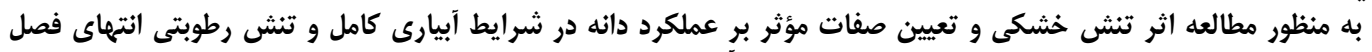

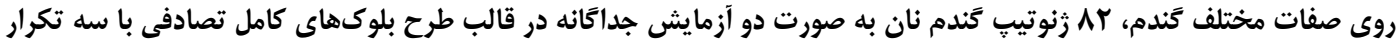

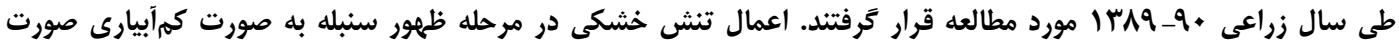

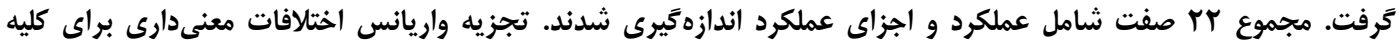

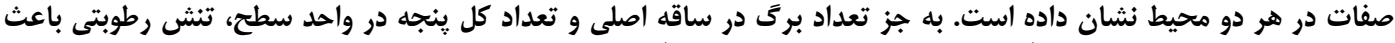

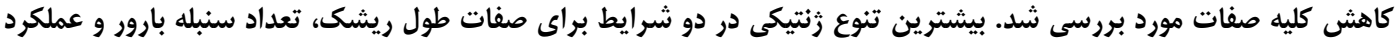

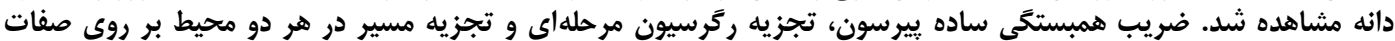

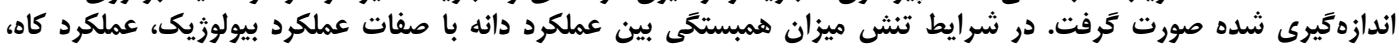

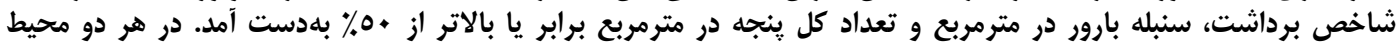

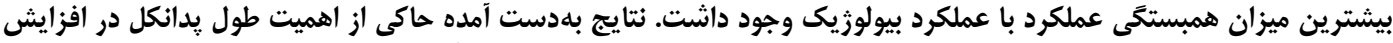

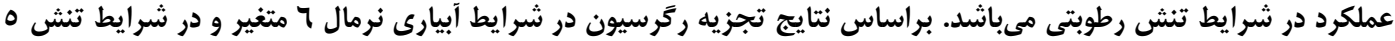

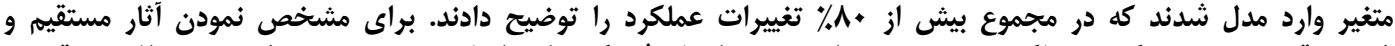

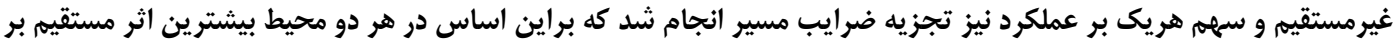

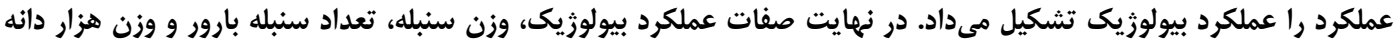

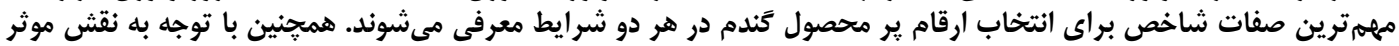

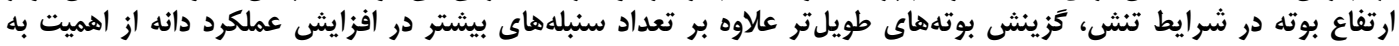

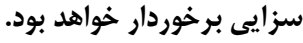

وازههاى كليدى: اجزاى عملكرد، تجزيه مسير، ركرسيون مرحلهاى، كمآبيارى، همبستكى

واكنشها در كياهان، از تغيير بيان ثن و متابوليسم سلول تان رئر

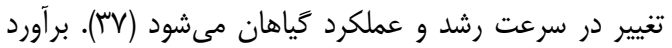

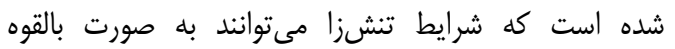

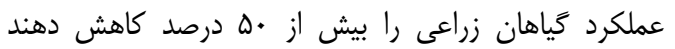
(FV)

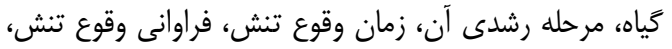

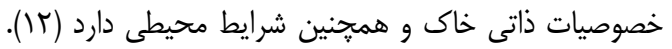

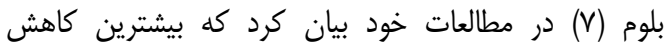

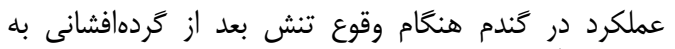

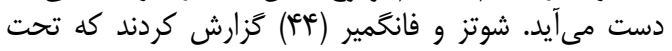

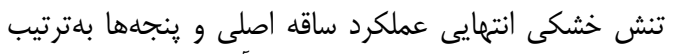

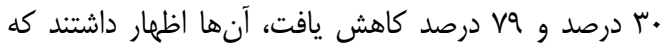
كاهش عملكرد بيشتر گندم تحت تنش كاهش عملكرد ينجهها است. كليك و و همكارد

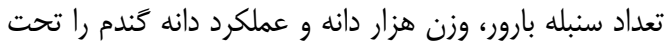

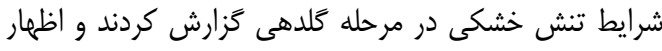

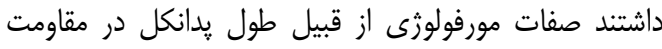

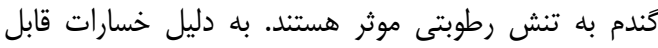

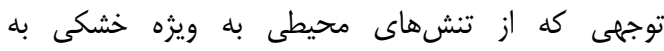

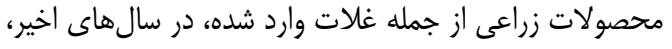

امروزه با توجه به روند رو به رشد جمعيت جهان و وند نياز روز

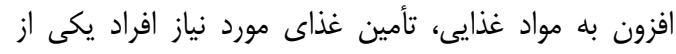

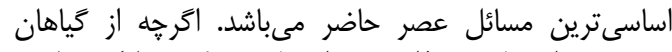

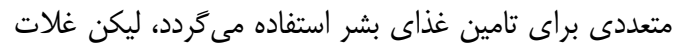

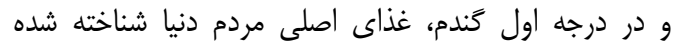

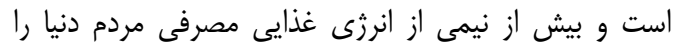

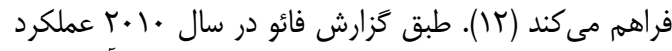

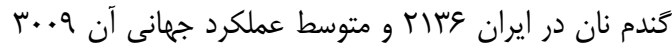

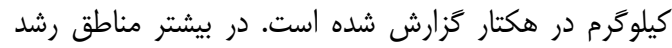

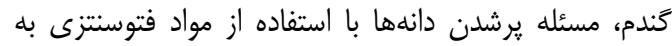

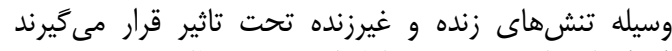

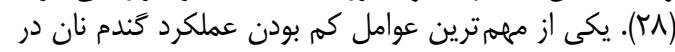

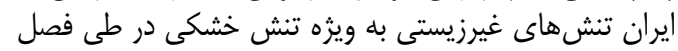

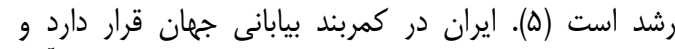

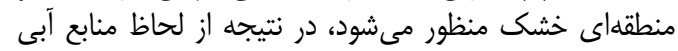

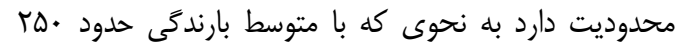

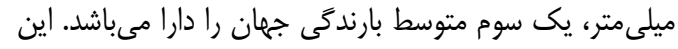

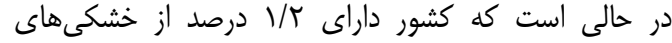

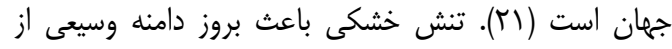


صفاتى است كه بيشترين اثر را بر عملكرد در شرايط نرمال و درائ

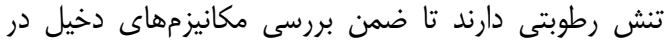

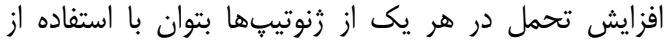

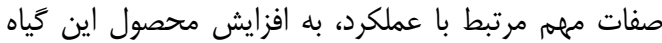

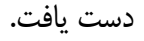

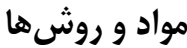

آزمايش در مزرعه تحقيقاتى برديس ابوريحان- دانشكان إناه

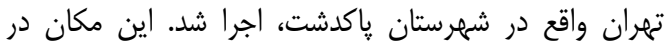

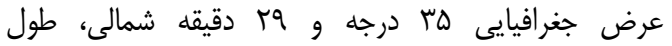

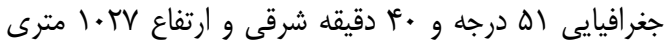

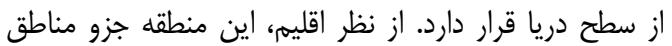

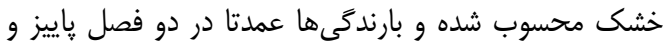

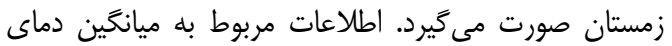

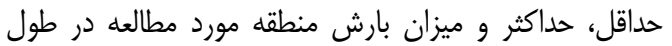

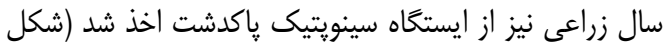

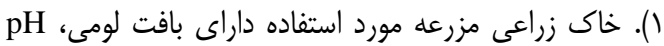

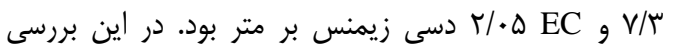

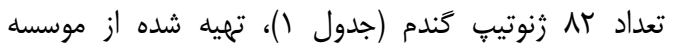

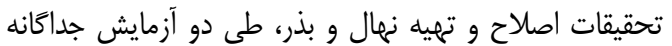

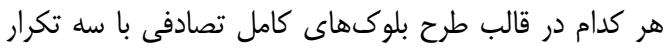

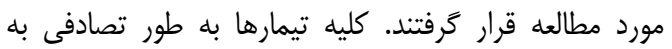

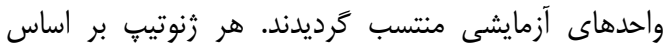

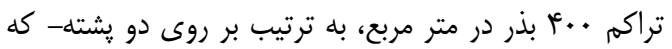

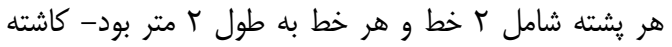

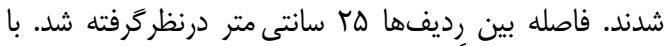

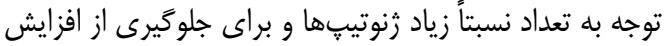

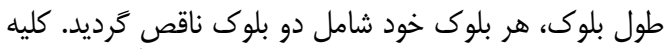

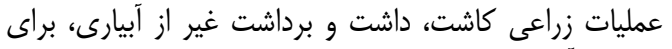

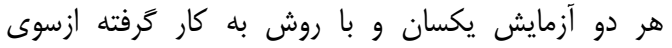

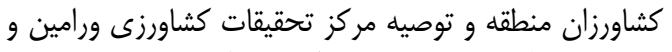

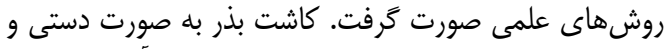

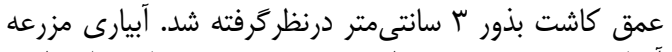

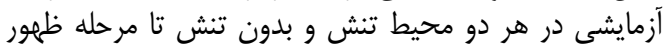

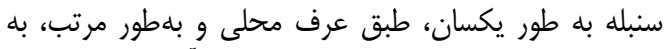

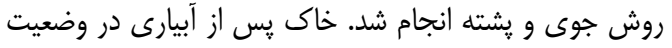

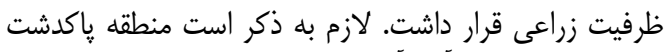

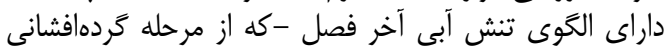

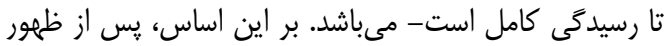

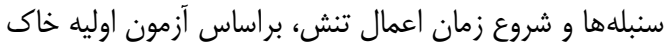

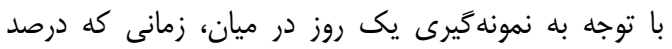

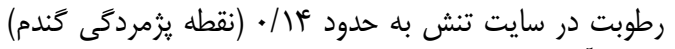

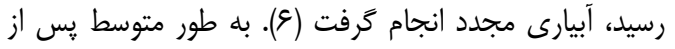

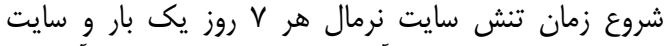

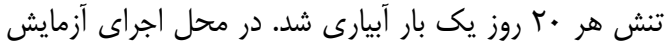

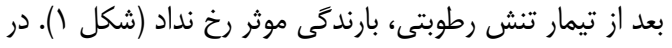

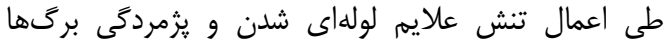

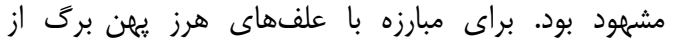
علفكش 2,4-D به ميزان ه/1/ ليتر در هكتار ماده تجارى در برد
بررسى واكنش كياهان زراعى به تنشهاى محيطى بسيار

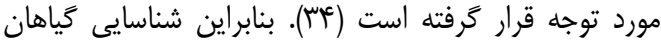

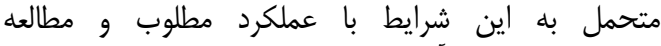

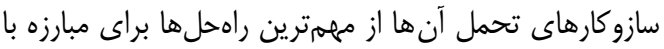

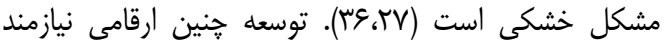

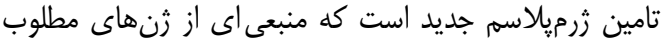

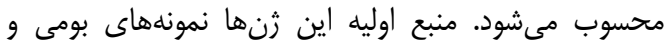

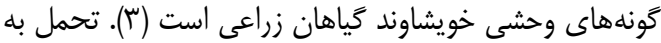

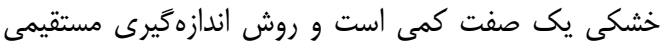

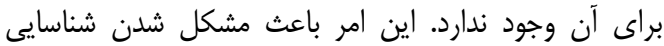

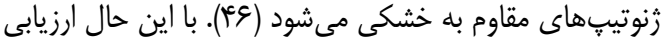

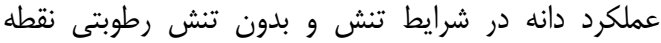

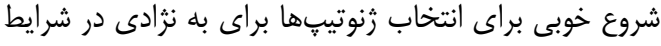

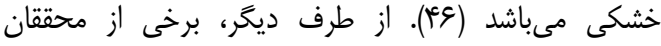

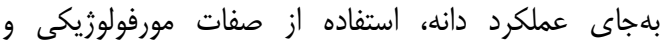

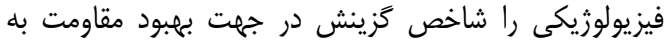

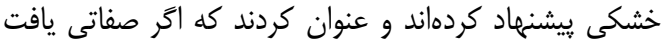

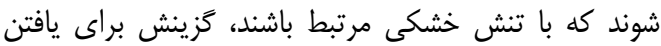

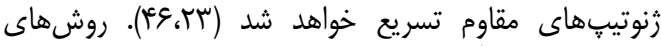

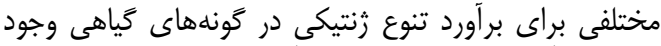

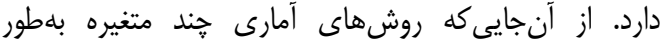

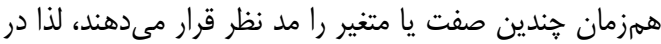

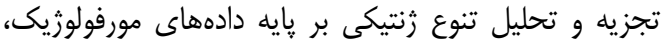

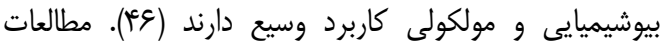
زيادى با استفاده از روشهاى مولى آمارى براى تعايع داريين نقش تنش

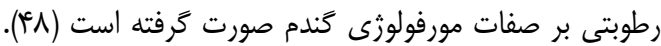

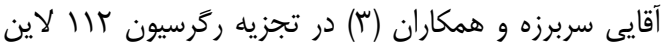

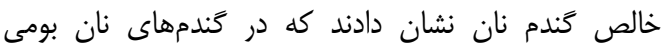

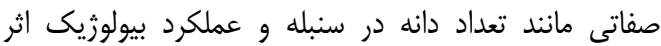

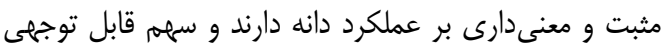

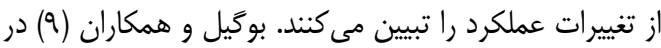
شرايط تنش خشكى همبستخى مثبت و و بسيار معنى دارى بنى بين

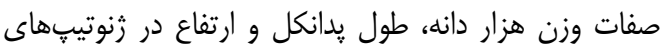

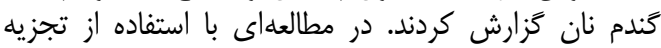

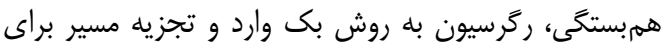

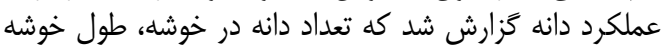

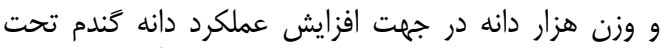

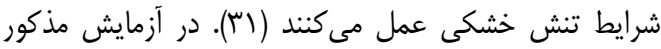

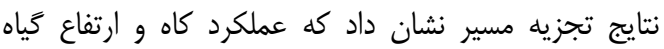

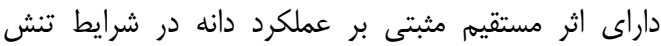

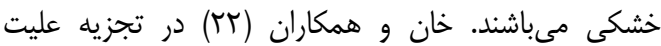

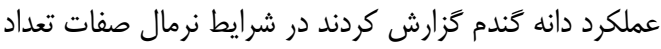

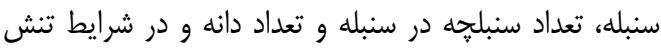

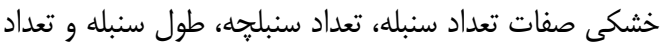

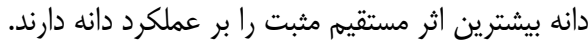

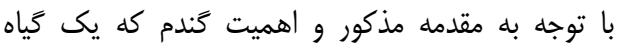

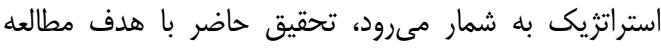

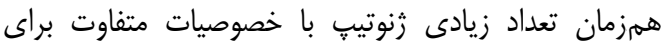
بررسى دقيقتر ارتباط بين بعضى صنات زني زراعى و شناسايى 


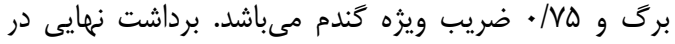

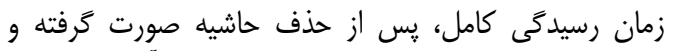

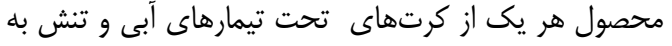

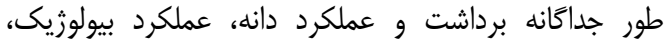
عملكرد كاه در واحد سطح، به عملكرد دانه دانه در واحد هكتانه

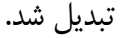

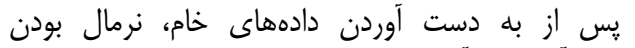

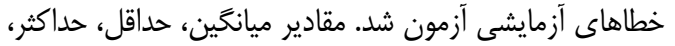

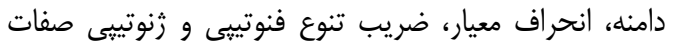

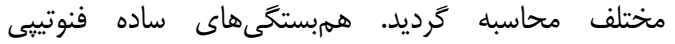

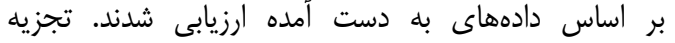

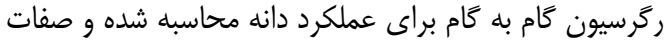

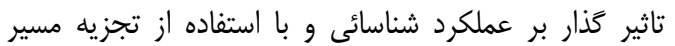

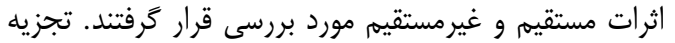

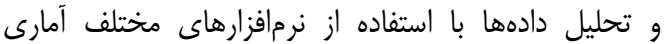

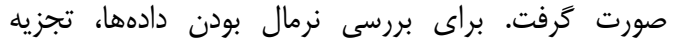

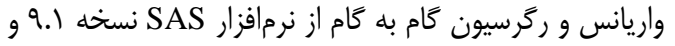

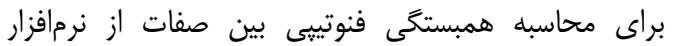

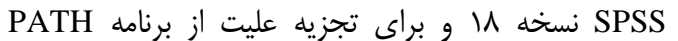

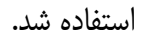

مرحله بِنجازنى استفاده شد، همرجنين وجين دستى در بهار

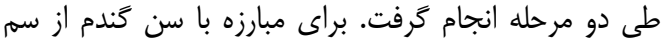

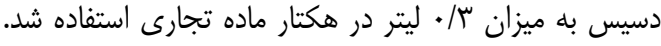

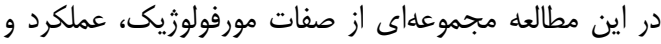

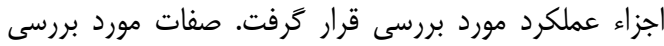

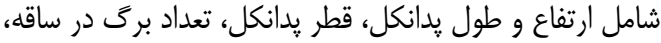

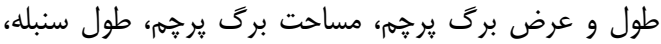

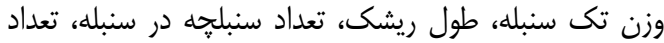

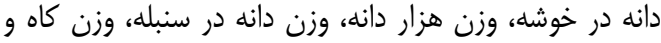

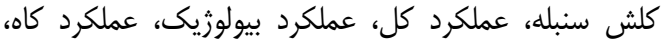

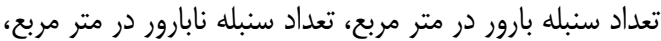

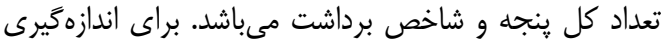

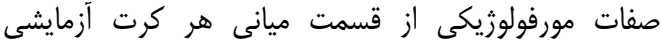

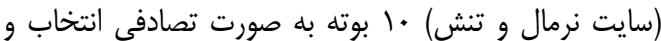

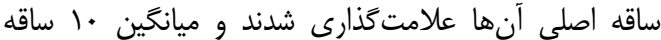

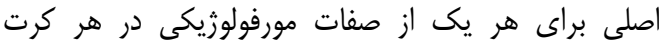

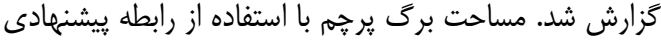

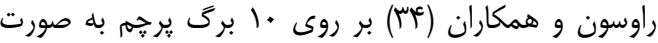
زير محاسبه شد:

$\mathrm{LA}=\mathrm{L} \times \mathrm{W} \times \cdot / \mathrm{VD}$

در اين رابطه L طول برگ، W عرض برگ، LA مساحت

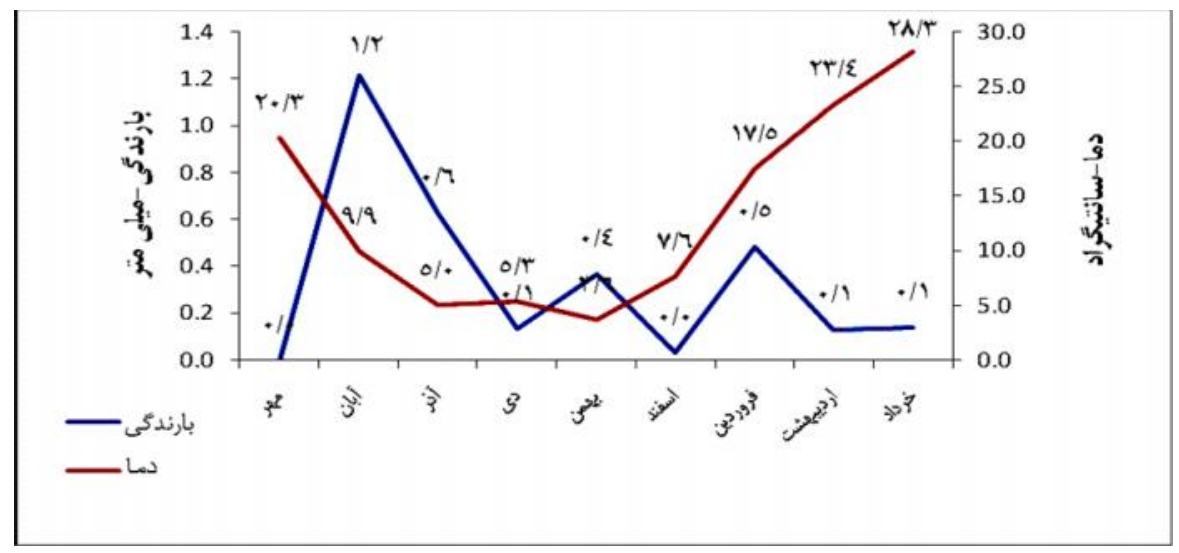

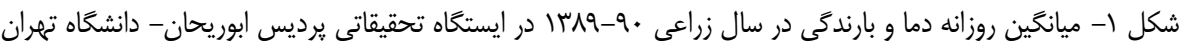
Figure 1. Average of temprature and precipitation in 2010 at research station of college of Aburaihan, UT. 
جدول ا- اسامى زنوتيڤهاى گَندم مورد بررسى در مطالعه تحمل به تنش رطوبتى آخر فصل Table 1. Evaluated wheat genotypes name in late season water stress assessment

\begin{tabular}{|c|c|c|c|c|c|c|c|}
\hline رنوتيّ & شماره & رنوتيبٍ & شماره & رنوتيّ & شماره & رنوتيٍّ & شماره \\
\hline تجن & $q^{\mu}$ & 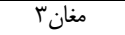 & Tr & 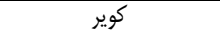 & Tr & los & 1 \\
\hline طوس & at & كرجr & if & شيرودى & זr & 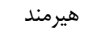 & $r$ \\
\hline شعله & 90 & Weebil & is & سبلان & re & j & r \\
\hline GR & 99 & جمران & is & كوهدشت & ra & بيشتاز & f \\
\hline MV-17 & sV & سردارى 1. & iv & كرج & re & اميد & $\Delta$ \\
\hline آذرا & 81 & مارون & is & ناز & tr & شاهِيسند & 8 \\
\hline بهار & 99 & unkhwon11 & 19 & الموت & rs & اروندموتانت & $\checkmark$ \\
\hline سايسون & v. & DN-11 & ه. & كاسيارد & rq & دريا & $\wedge$ \\
\hline سيستان & $n$ & WS-82-9 & Q) & 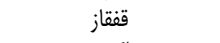 & r. & مرودشت & 9 \\
\hline 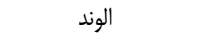 & VT & البرز & $\Delta T$ & زاكرس & M & اكبرى & 1. \\
\hline مهدوى | مي & $v^{\mu}$ & كاسكوثن & QI & 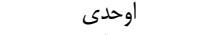 & r & 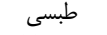 & 11 \\
\hline سومالى؟ & $v^{e}$ & 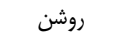 & $\Delta F$ & تيييك & זי & بزوستايا & Ir \\
\hline سردارى & VQ & GS & $\Delta \Delta$ & اكسكالبور & re & 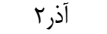 & זו \\
\hline اترك & ve & ق ق قدس & $\Delta S$ & S-83-3 & ra & آرتا آ & if \\
\hline 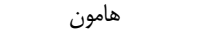 & w & زرين & $\Delta V$ & كرخه & rq & رسول & 10 \\
\hline كراس فلات هامون & vu & شاهى & $\Delta \wedge$ & نويد ب لميد & rV & آزادى & 18 \\
\hline بيات & va & كراس البرز & $\Delta q$ & مغان & r & سياهان & iv \\
\hline بولانى & ᄉ. & 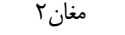 & 9. & ن نيك نزاد & "q & فرونتانا & 11 \\
\hline بكى كراس روشن بهاره & 1) & رصد & 8) & بكى كراس روشن زمستانه & f. & شيراز & 19 \\
\hline فونگ & $\Lambda T$ & استار & st & داراب & il & اينياء & r. \\
\hline & & & & 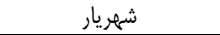 & et & ويرى ناك & r \\
\hline
\end{tabular}

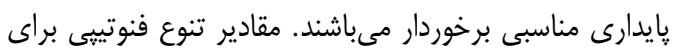

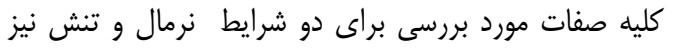

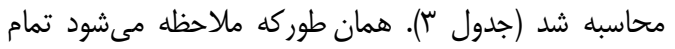

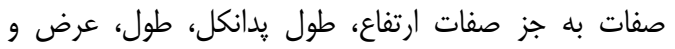

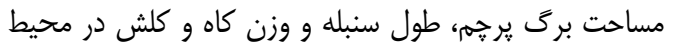

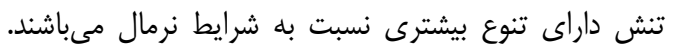

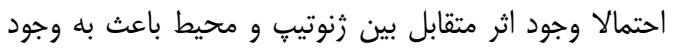

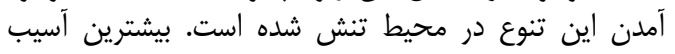

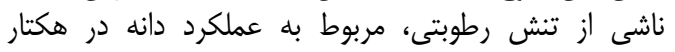

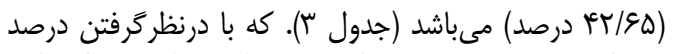

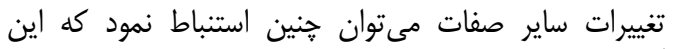

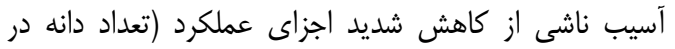

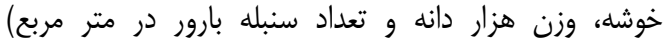

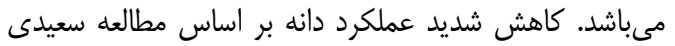

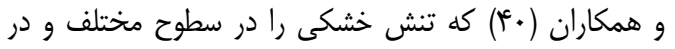

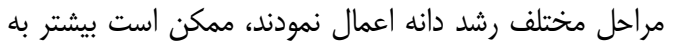

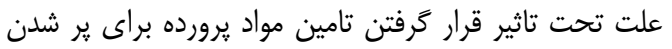

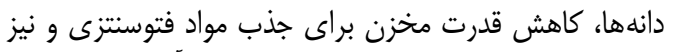

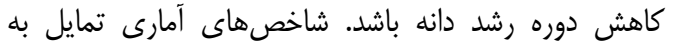

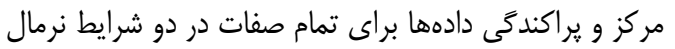

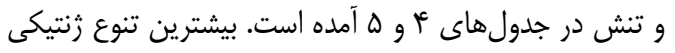

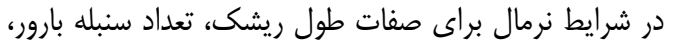

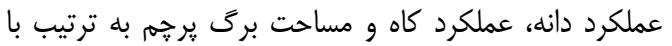

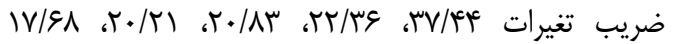
مى باشد.

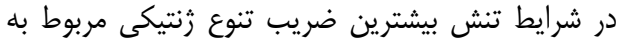

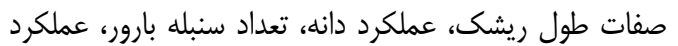

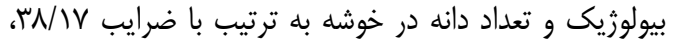

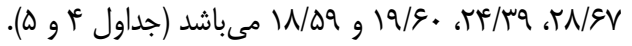

\section{نتايح و بحث}

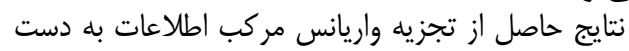

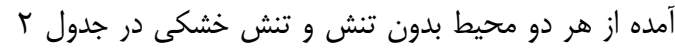

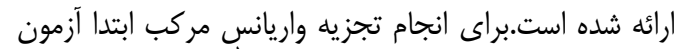

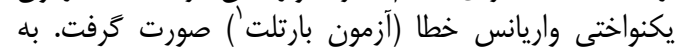

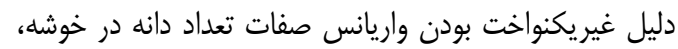

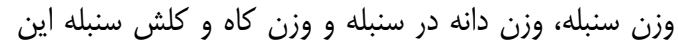

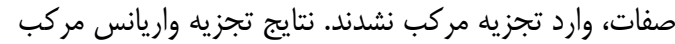

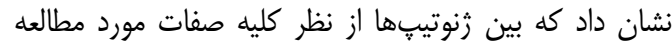

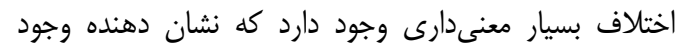

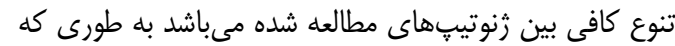

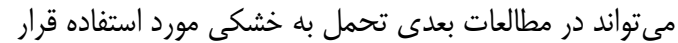

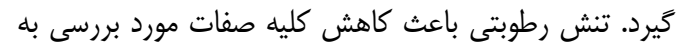

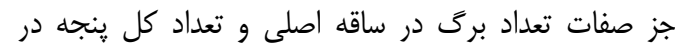

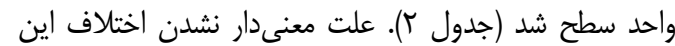

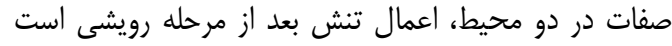

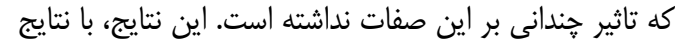

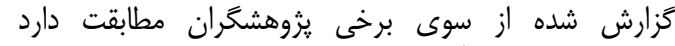
×

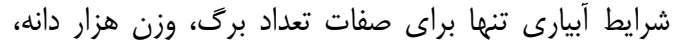

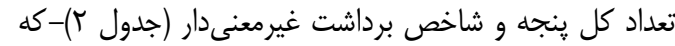

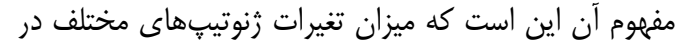

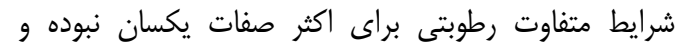

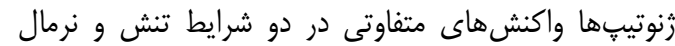

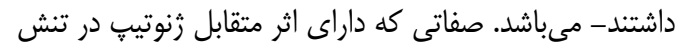

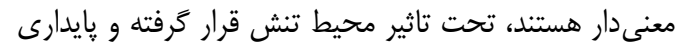

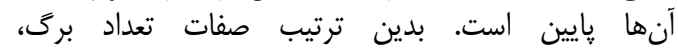

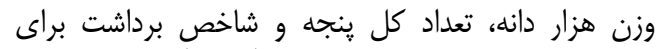
اصلاح عملكرد در هر دو شرايط تنش آبى و آبيارى طبيعى از بردائ 
جدول r- تجزيه واريانس مركب (ميانخين مربعات) بين صفات مختلف زنوتيقهاى گندم تحت هر دو شرايط آبيارى نرمال و تنش رطوبتى Table 2. Combined analysis variance (mean squares) among different tratits in wheat genotypes under both normal

\begin{tabular}{|c|c|c|c|c|c|c|c|}
\hline عرض برى & طول برى & $\begin{array}{c}\text { برك ساقه } \\
\text { بلى }\end{array}$ & بدانكل & طول يدانكل & ساقه اصلى & آزادى & تغنبير \\
\hline$\cdot /$ $/$ Q४ & rqv/qu & g/ATE-rns & $\cdot / N F D^{*}$ & $\Delta \cdot 9 \Delta / V q^{9+7}$ & $1901 / 9 \Lambda^{*}$ & 1 & شرايط آبيارى \\
\hline.$/ \cdot 1 f a^{* *}$ & $10 / 9 \mu^{* * *}$ & $\cdot /$ rorV & $\cdot|\cdot| Q r^{* * *}$ & $r \eta / / r^{* * *}$ & 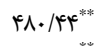 & r & تكرار درون محيط \\
\hline$\cdot / \cdot \Delta \Delta \Gamma^{* * *}$ & $19 / M^{* *}$ & $.19199^{* *}$ & $\cdot / \cdot \Delta f^{* * *}$ & $V Q / T^{* * *}$ & $r W / / \varphi^{* * *}$ & 1) & رنوتيٍّ \\
\hline$\cdot / .+\mu \varphi^{* * *}$ & $f /\left.f\right|^{* *}$ & $\cdot /{ }^{\mathrm{ns}}$ & $\cdot / \cdot .1^{* *}$ & $\mathrm{~N} / \mathrm{q}^{* *}$ & $\Delta F / V \Lambda^{* *}$ & $\wedge$ & اثر متقابل زنوتيڤ در شرايط آبيارى \\
\hline 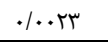 & $1 / T V$ & .1. .91 & $\cdot 1 \cdots \infty$ & S/AT & TI/Tr & Trte & اشتباه آزمايشى \\
\hline
\end{tabular}

Continued table 2

\begin{tabular}{|c|c|c|c|c|c|c|c|}
\hline تعارو سنبله & وزن هزار دانه & طول ريشك & تعداد سنبلجه & طول سنبله & مساحت برى & آزادى & تغنيير \\
\hline Ir. rQgN/द** & DF9N/91* & $\mu N / \cdot D^{* * *}$ & $1 \cdot 1 / /^{\text {t* }}$ & $\Lambda \mathrm{N} / \mathrm{r}^{* *}$ & $\operatorname{rog} / / \mathrm{R}^{\mathrm{F*}}$ & 1 & شرايط آبيارى \\
\hline $\mid \operatorname{s\varphi } \cdot \Delta / /^{* *}$ & $|9| / \mathrm{Ve}^{* * *}$ & $\cdot / \pi v \cdot \Lambda^{*}$ & $1 / 1 r^{* *}$ & $r / I^{* *}$ & $19 / 19^{* *}$ & f & تكرار درون محيط \\
\hline $91+4 \times 9 / 19 *$ & $199 / 9 t^{* * *}$ & $\mathrm{rN} / \mathrm{r}^{* * *}$ & $1 \cdot 19^{* * *}$ & $r / 4 e^{* * *}$ & $r M / \mu I^{* * *}$ & 1) & رُنوتيب \\
\hline $1 . .9 \% / 0^{*}$ & $|f / \mu|^{\mathrm{ns}}$ & $\cdot / T \Delta \Delta Q^{*}$ & $\cdot / T V^{* * *}$ & 每/ & $r / \kappa r^{* * *}$ & 1) & اثرمتقابِل زنوتيِ در شرايط آبيارى \\
\hline VTNE/V & $11 / \uparrow^{\infty} \Delta$ & . MIfF & •/NALT & - /FAT & $1 / \mu$ & MTE & اشتباه آزمايشى \\
\hline
\end{tabular}

Continued table 2

\begin{tabular}{|c|c|c|c|c|c|c|c|}
\hline برداشت & عملكرد كاه & بيولوزيك & كلكرد & تعداد كل ينجه & تعداد سنبله & آزادى & تغنيب \\
\hline$\Delta F^{F} / 9 Q^{* *}$ & $\Delta T \Delta / \cdot r^{* * *}$ & TMFY/IS & $8 / V e^{e^{n+m}}$ & ISATY $/ \Delta^{\text {ns }}$ & FVTFED/F" & 1 & شرايط أبيارى \\
\hline $9 \pi / T^{*}$ & $\mid r 1^{* * *}$ & $q \Psi / \mathscr{q} \Psi^{* * *}$ & $9 / F V^{* *}$ & $V V G \cdot r / q^{* * *}$ & $\operatorname{rr\Delta V} / \Lambda I^{* * *}$ & r & تكرار درون محيط \\
\hline $19 \mathrm{~V} / \mathrm{re}^{* * *}$ & $\mid r / \cdot r^{* *}$ & $r N / \varphi^{* * *}$ & $g / V e^{* * *}$ & $11 \cdots \Delta / r \Delta^{* *}$ & MTIF/FQ"** & 1) & رنوتيب \\
\hline$r r / g T^{n s}$ & $\Gamma / \Lambda \Delta^{* * *}$ & $V / N r^{* * *}$ & $1 / / f^{*}$ & $11 \Delta \Lambda \cdot / 8^{\mathrm{ns}}$ & $|\varepsilon \cdot 9 / \mu|^{*}$ & $\wedge$ & اثرمتقابل زنوتيّ در شرايط آبيارى \\
\hline $19 / 91$ & $T / M I$ & $r / \mathrm{V}$ & . VNAN & $94 \cdot 9 / 4 \pi$ & $1.91 / .1$ & MTf & اشتباه آزمايشى \\
\hline
\end{tabular}

جدول بـ- درصد تغييرات ناشى از تنش رطوبتى بر روى صفات اندازهيرى شده زنوتيِهاى گندم Table 3. Change rate of measured traits on wheat genotypes affected by water stress

\begin{tabular}{|c|c|c|c|c|c|}
\hline \multirow[t]{2}{*}{ درصد كاهش صفات } & \multicolumn{2}{|c|}{ ضريب تنوع فنوتييى صفت (٪) } & \multirow[t]{2}{*}{ ميانكين صفات در } & \multirow[t]{2}{*}{ ميانكين صفات در شرايط } & \multirow[t]{2}{*}{ صفات } \\
\hline & شرايط تنش & شرايط نرمال & & & \\
\hline$-\mid 1 / / \mu$ & $9 / 99$ & $I T / \Lambda Q$ & $9 \mathrm{~V} / \cdot 1$ & VQ/GK & رتفاع (سانتىمتر) \\
\hline$-19 / 0$. & 1.199 & $\mid F / 4$ & $r \varphi / \Delta Q$ & $r T / q \Lambda$ & طول يدانكل (سانتىمتر) \\
\hline$-11 / 9 \Lambda$ & $1 . / \% \omega$ & $q / \Psi r$ & . & $\cdot / \pi \Delta \mid$ & تطر بِدانكل (سانتىمتر) \\
\hline$+1 / \mu$ & $V / 4 F$ & $V / F$ & $f|q|$ & $F / \Delta \Delta$ & نعداد برى \\
\hline$-\mid Y / \cdot r$ & $|r /| \varphi$ & שr/F & $1 \% / 09$ & $\mid \omega / \digamma A Q$ & طول برَّ بر گمى (سانتىمتر) \\
\hline$-F / V$ & $q / \Psi q$ & 1.1 .4 & 1 & $1 / \cdot 0$ & عرض برك بِرجِم (سانتىمتر) \\
\hline$-\mid \varepsilon / 19$ & $\mid V / M f$ & $19 / .9$ & $1 . / T V$ & $\mid r / T \Delta$ & مساحت برى برِمٍ (سانتىمتر) \\
\hline$-V / V T$ & N/F & $q / r)$ & $q / \Delta \rho$ & 1.148 & طول سنبله (سانتىمتر) \\
\hline$-1 N / 90$ & $181 \cdot V$ & $1 \% / \Lambda$ & $r / \cdot 1$ & $r / \uparrow \Lambda$ & وزن تك سنبله اصلى (كرم) \\
\hline$-9 / \Delta$ & ו ו & $\mathrm{TV} / \boldsymbol{\varphi}$. & سז & 9 & طول ريشك (سانتىمتر) \\
\hline$-\Delta / \Gamma$ & $N / N F$ & $N / F^{e}$ & $19 / T \Delta$ & $I V / / Q$ & نعداد سنبلجه در سنبله \\
\hline$-19 / r$. & $I V / r A$ & $19 / 9$. & TN/DT & I I I I & نعداد دانه در خوشه \\
\hline$-\mid Q / \not V$ & $19 / \mathrm{AV}$ & $\mid N / 48$ & $r \Delta / \& \Lambda$ & FT/KI & تعداد سنبله بارور (مترمربع) \\
\hline$-r \mid / \& q$ & $1 / 49$ & $18 / 99$ & $1 / \% v$ & $1 / V F \Delta$ & تعداد سنبله غيربارور (مترمربع) \\
\hline$-1 N / r 9$ & $r g / T E$ & $r E / \Delta V$ & $F \Delta N / \mu F$ & $\Delta 9 \cdot / 9 T$ & تعداد ينجه (متر مربع) \\
\hline$+4 t / Q V$ & $14 / 19$ & Tr & TAT/\&S & TIN/FT & وزن هزار دانه (َّرم) \\
\hline$-r / A r$ & $M / M$ & W/A & $V F i / g r$ & $V W Q / r \Delta$ & عملكرد كل (تن/ هكتار) \\
\hline$-r r / A l$ & tr/Tr & $r \cdot / \Lambda \varepsilon$ & N/aV & 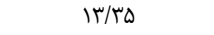 & عملكرد بيولوزيك (تن/ هكتار) \\
\hline$-r \Delta / Q \Delta$ & $r / \Delta S$ & $r F / M$ & $\Delta / \wedge \Delta$ & $V / q$. & وزن كاه و كلش (تن/ هكتار) \\
\hline$-19 / 49$ & $\mid \Delta / \Delta \Delta$ & $\mid E / T V$ & $\mu E / \mu F$ & FI/T & شاخص برداشت (٪) \\
\hline$-F T / 9 D$ & rT/Vg & 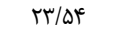 & $r / M r$ & $\Delta / A^{\epsilon}$ & وزن دانه در تك سنبله (كرم) \\
\hline
\end{tabular}


جدول ז- شاخصهاى آمارى تمايل به مركز و براكندگى صفات زراعى- مورفولوزيكى (شرايط نرمال) در زنوتيبهاى گندم مورد بررسى Table 4. Statistics parameters of agro-morphological traits of wheat genotypes in normal condition

\begin{tabular}{|c|c|c|c|c|c|c|c|c|}
\hline 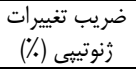 & انحراف & ميانخين & 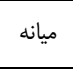 & 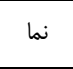 & كمينه & بيشينه & دامنه & صفات \\
\hline $1 T / r$ & $\mid 1 / \pi 1$ & $V \Delta / \&$ T & $V T / \& D$ & $V \cdot$ & $\Delta \Delta / V$ & $11 \cdot / \Delta \mathrm{V}$ & $\Delta F / \wedge V$ & ارتفاع (سانتىمتر) \\
\hline $\mid r / \uparrow \wedge$ & Q/DT & $\mathrm{rr} / \mathrm{Q \Lambda}$ & r. & r l & 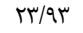 & 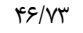 & rt/A & طول پيدانكل (سانتىمتر) \\
\hline$\Lambda / \cdot \Lambda$ & 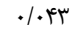 & $\cdot|r \Delta|$ & $\cdot / r \Delta \cdot$ & 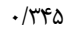 & $\cdot / T V$ & $\cdot / F \Delta$ & $\cdot / 1$ & قطر يدانكل (سانتىمتر) \\
\hline $\mathrm{V} / \mathrm{MI}$ & • & $\uparrow / \Delta \Delta$ & $r / \Delta$ & $\Delta$ & $r / q$ & $\Delta / \digamma$ & $1 / \Delta$ & تعداد برى \\
\hline IT/Tr & $r / \Delta V$ & $1 \omega / \uparrow \omega$ & W/D/M & 10 & 1.194 & 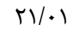 & $1 \cdot / r V$ & طول برگ ير خهم (سانتىمتر) \\
\hline $9 / 8 \wedge$ &.$/ 11 f$ & $1 / \cdot \Delta r$ & $1 / \cdot f$ & 1 & $\cdot / M$ & $1 / F V$ & .109 & عرض برى يرجهم (سانتىمتر) \\
\hline $\mid V / 9 \Lambda$ & $r / V \cdot$ & $I T / T \Delta$ & $11 / \mathrm{M}$ & $1.1 \cdot 1$ & $V / \Delta F$ & $19 / 14$ & $11 / \Delta \Lambda$ & مساحت برگ ير پهم (سانتىمتر) \\
\hline К/99 & $1 / \cdot 1$ & (1. 1. & $1 \cdot / \pi$ & 1. & $V / \& \Lambda$ & IT/VT & $\Delta / \cdot \mathcal{F}^{\mathrm{C}}$ & طول سنبله (سانتىمتر) \\
\hline $1 \% / 1$. & $\cdot / \leftarrow \wedge$. & $r / F \wedge$ & $r / 4 q$ & r & $1 / 91$ & $r / 9 \Delta$ & $r / \cdot r$ & وزن تك سنبله اصلى (گرم) \\
\hline$r V /$ tef & r/rq & $\varepsilon$ & $\varepsilon / 48$ & $\checkmark$ & ש & $1 r / r q$ & $\mid r / 19$ & طول ريشك (سانتى متر) \\
\hline$V / \cdot 9$ & $1 / F+$ & $I V / I Q$ & $I V / F$. & $I V / \Delta$. & $1 \% / 48$ & $r \cdot / l$ & $9 / 9 t$ & تعداد سنبلهِ در سنبله \\
\hline $18 / 91$ & $9 / 4 q$ & $F+/ T$ & $f 1 / 1$. & rV & rt/qu & $9 \cdot / 1 \pi$ & $r V / T$ & تعداد دانه در خوشه \\
\hline שמ/rt & $\mid \& M / T \Delta$ & $\Delta G \cdot / 9$ & DTV & $f+$. & req & $\| F \varepsilon / \pi$ & $\vee q \vee / r$ & تعداد سنبله بارور (مترمربع) \\
\hline $8 / r q$ & $M F / T I$ & TIN/FT & rit & $r+$. & $1 \% q / 9$ & $r v \cdot$ & $1 \% \cdot / f$ & تعداد سنبله غيربارور (مترمربع) \\
\hline $\mid \& / \Delta S$ & $I V E / F V$ & $V \vee q / r \Delta$ & VDF & $9 \Delta \Lambda$ & DIF/\&9 & $\| T \Delta / / 9$ & Nrg/af & تعداد هنجه (متر مربع) \\
\hline $\mid Q / \Delta V$ & $g / V^{e}$ & 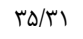 & 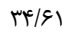 & $r \& / Q$. & IV/TF & $\Delta 1 / T$. & $m \varphi / .9$ & وزن هزار دانه (كرم) \\
\hline$r \cdot / \Lambda \mu$ & $1 / \Delta \Lambda$ & $\Delta / F F$ & $\Delta / \Gamma \cdot$ & $f / f$ & $r / M$ & N/TV & $\Delta / f$ & عملكرد كل (تن/ هكتار) \\
\hline IV/G & $r / v \cdot$ & 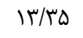 & $15 / 19$ & $1 \cdot / \pi$ & $\Lambda / \mathrm{N}$ & $r \cdot / / f$ & 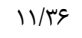 & عملكرد بيولوزيك (تن/ هكتار) \\
\hline$r \cdot / 4$ & $T / \Delta S$ & $V / q$. & $V / f$ & - & $\Gamma / \Delta \Lambda$ & $\mid r / 8 \Lambda$ & $1 \cdot / 1$ & وزن كاه و كلش (تن/ هكتار) \\
\hline $1 \% / 94$ & $\mathrm{~V} / \cdot \mathrm{r}$ & $f / / t$ & $f i / f a$ & - & re/M & $\Delta q / V$ & Tr/Ar & شاخص برداشت (\%) \\
\hline س & $\cdot / 4 \cdot t$ & I/VFa & I/Va & $1 / 9$. & $1 / \cdot 1$ & $r / 9 q$ & $1 / 81$ & وزن دانه در تك سنبله (كرم) \\
\hline
\end{tabular}

جدول ه- شاخصهاى آمارى تمايل به مركز و براكندگى صفات زراعى- مورفولوزيكى (شرايط تنش رطوبتى) در زنوتيبهاى گندم مورد بررسى . Table 5. Statistics parameters of agro-morphological traits of wheat genotypes under water stress condition

\begin{tabular}{|c|c|c|c|c|c|c|c|c|}
\hline ضريب تغييرات زنوتييى & انحراف & ميانخين & 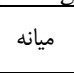 & نما & كمينه & بيشينه & دامنه & صفات \\
\hline$\wedge / \wedge \varepsilon$ & $\Lambda / \Lambda \Lambda$ & $9 \mathrm{~V} / \cdot 1$ & $g \varepsilon / T^{\circ}$. & V. & $\Delta F / \&$ & 19/\% & 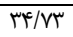 & ارتفاع (سانتىمتر) \\
\hline $9 / 8 \wedge$ & $r / v r$ & $r \& / \Delta D$ & $r \varepsilon / T$. & $r Y / r$. & 19/Dr & TH/f & $I r / \wedge V$ & طول پيدانكل (سانتى متر) \\
\hline 1. & נמש:/. & | & هוm & $\cdot / \mu \cdot$ & $\cdot|r|$ & . At & $\cdot|r|$ & قطر پِانكل (سانتىمتر) \\
\hline $\mathrm{V} / \mathrm{MI}$ & س & $f / \Delta \Delta$ & $r / \Delta$ & $\Delta$ & $r / q$ & D/TG & 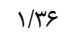 & تعداد برى \\
\hline $1 r / 4 q$ & $1 / 91$ & $1 \% / 09$ & 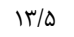 & it & $9 / 19$ & $I V / \Lambda \Delta$ & V/aq & طول برى يرجم (سانتىمتر) \\
\hline N/ᄉ & $\cdot / 1 \cdot 1$ & 1 & 1 & 1 & $\cdot / \Lambda F$ & 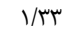 & $\cdot / 19 q$ & عرض برى ير جهم (سانتىمتر) \\
\hline $18 / 1$. & 1/9V & $1 \cdot / T V$ & 1./ra & $1 . / 1 \mathrm{~V}$ & $\mathrm{~V} / \cdot \mathrm{t}$ & $1 f / 9$. & $\mathrm{V} / \Delta \mathrm{\Lambda}$ & مساحت برگ يرجهم (سانتىمتر) \\
\hline V/99 & ./AVT & $Q / \Delta \varphi$ & $9 / \Delta$ & 9 & $\checkmark$ & سז/11 & سז/ & طول سنبله (سانتىمتر) \\
\hline$|f / 9|$ & ERE & $r / \cdot 1$ & $1 / 98$ & r & $1 / \pi$ & t/AT & $1 / \Delta 1$ & وزن تك سنبله اصلى (گرم) \\
\hline HN/IV & $r / 1$. & س & 8 & 9 &.$/ 19$ & $1 . / 1 \mathrm{~V}$ & $1 \cdot / \mathrm{N}$ & طول ريشك (سانتىمتر) \\
\hline$N / \cdot 1$ & $1 / r V$ & $18 / T \Delta$ & $19 / 0$ & IV & ש & IN/VE & $g / f$ & تعداد سنبلهه در سنبله \\
\hline $1 N / 09$ & $N / \Delta$ & $r \omega / s \Lambda$ & $\mathrm{r} \omega / \mathrm{q}$ & rv & $r \cdot / \Delta$ & $\Delta F / r$ & 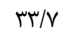 & تعداد دانه در خوشه \\
\hline$r ז /$ qq & 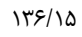 & $F \Delta N / F F$ & שrץ & שמת & פשr & $9 M$ & $v \neq q$ & تعداد سنبله بارور (متر مربع) \\
\hline N/9T & ( ) & TAT/ES & rVq & $r \Delta \Delta$ & $194 / \pi$ & rqه/r & $r \cdot 1$ & تعداد سنبله غيربارور (متر مربع) \\
\hline $\mid s / 48$ & $10 \xi / \mu$. & $V F I / A T$ & Vet & $9 \cdot 1$ & IED & $|K| F / M$ & $V \in q / r$ & تعداد ينجه (متر مربع) \\
\hline 181.9 & $\Delta / \mathrm{q}$ & TN/DT & $r V / \Lambda$ & $r \Delta / \Lambda$ & $\mid \Delta / \& \Delta$ & $F \mid / r$ & $r \Delta / V T$ & وزن هزار دانه (كرم) \\
\hline TN/FV & $1 / 10$ & m/lt & r/ar & r & $1 / r V$ & $\varepsilon / \cdot \Delta$ & $f / \varepsilon \Lambda$ & عملكرد كل (تن/ هكتار) \\
\hline $19 / 9$. & $T / E V$ & N/qV & N/VT & $\mathrm{V} / \mathrm{M}$ & 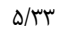 & $\mid \mp / \& \Lambda$ & $9 / 10$ & عملكرد بيولوزيك (تن/ هكتار) \\
\hline $1 \mathrm{~V} / \mathrm{a}$. & $1 / \Delta \varphi$ & $\Delta / \wedge \Delta$ & $\Delta / V I$ & - & T/ט/ & $q / \cdot r$ & $\Delta / \& q$ & وزن كاه و كلش (تن/ هكتار) \\
\hline $14 / 99$ & $8 / 48$ & Tr/me & Tr/VT & - & TI/VQ & $f \Delta / r$. & rT/Fa & شاخص برداشت (\%) \\
\hline $15 / 91$ & - /Tar & $1 / T V$ & 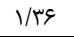 & $1 / 9$ & $\cdot / 79$ & $1 / 94$ & $1 / 10$ & وزن دانه در تك سنبله (كرم) \\
\hline
\end{tabular}


طرفى به دليل دارا بودن همبستخى مثبت ميان ارتفاع كَياه و

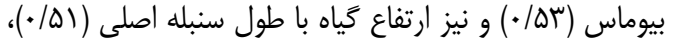

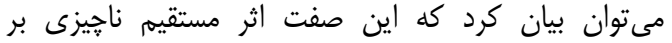

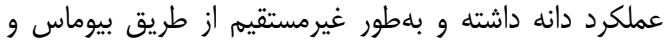

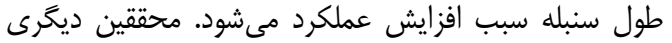

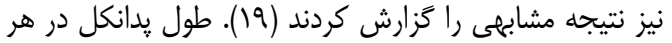

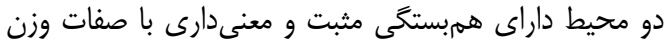

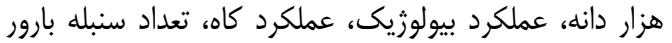

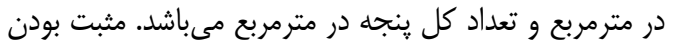

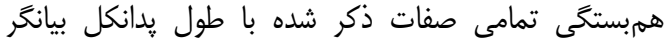

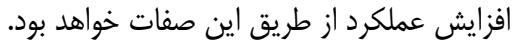

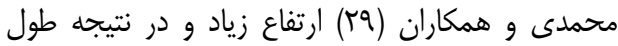

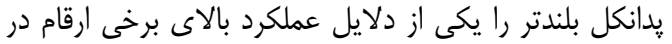

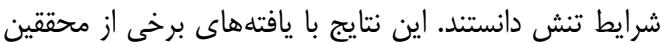

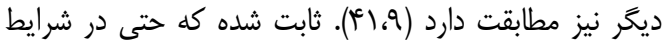

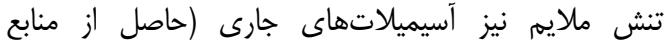

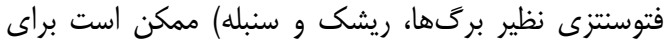
يرشدن دانه كافى نباشد (هان (1).

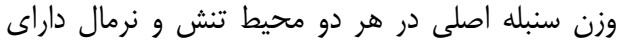

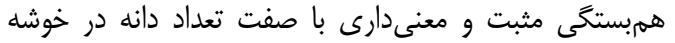

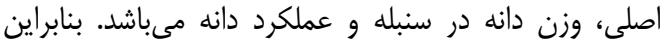
مىتوان اين طور بيان كرد كه با افزايش تعداد دانه در خوشئه دئه

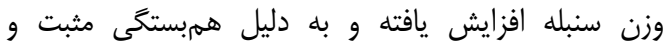

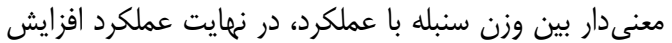

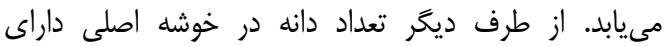

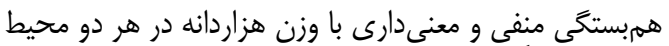

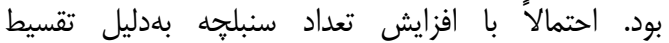

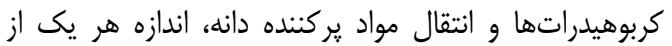

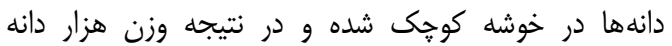

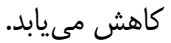
با وجود اين برخى محققان معابد متتقدند كه تعداد دانه

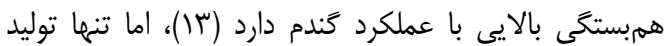

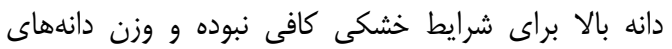

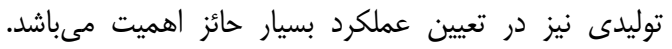

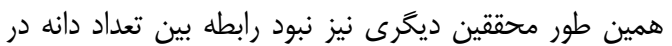

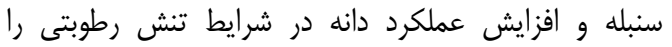

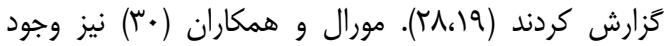

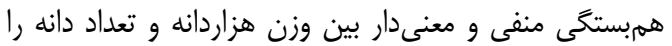

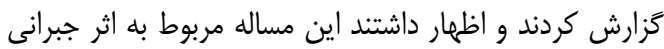

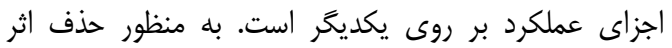

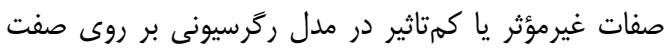

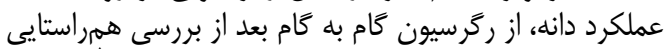

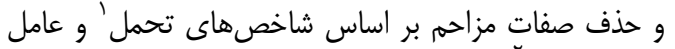
تورم واريانس ' استفاده شد (جداول ^رو و ج).

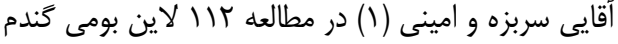

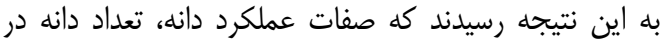

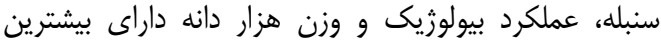

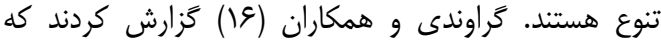

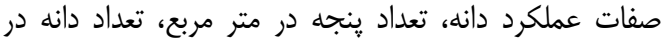

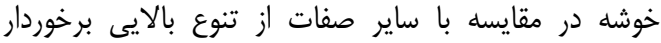

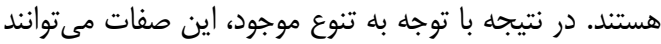

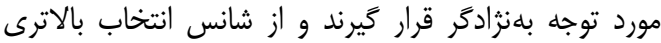

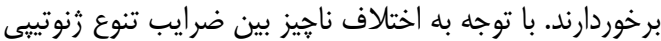

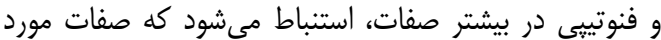
بررسى كمتر تحت تاثير محيط قرار كر فتنهاند. در هر دو دو شرايط

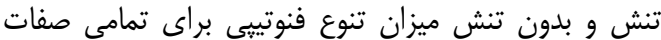

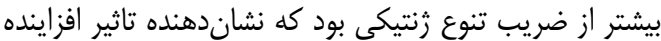

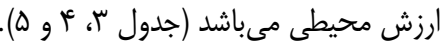

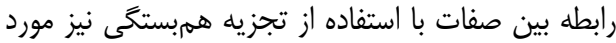

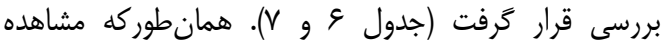

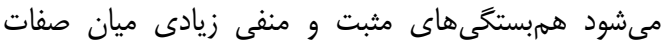

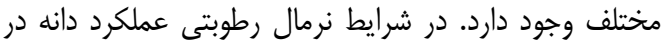

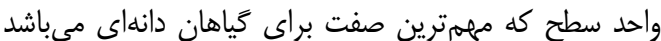

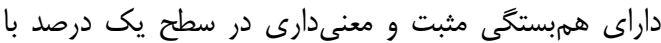

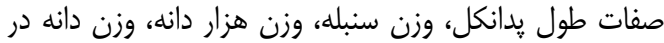

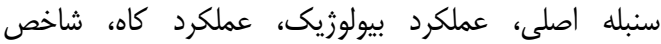

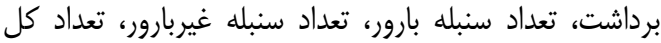

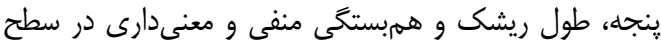

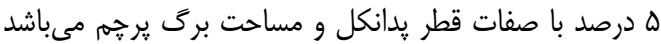

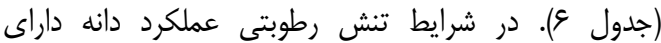

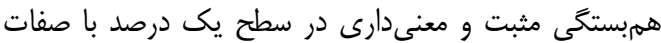

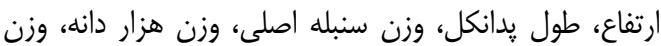

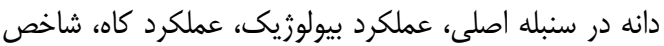

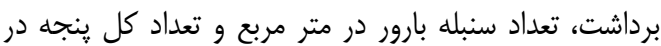

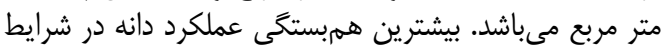

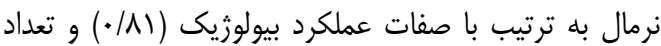

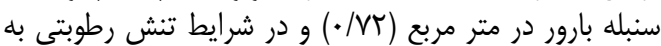

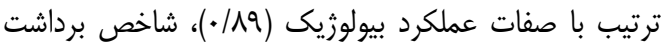

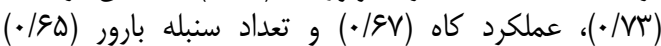

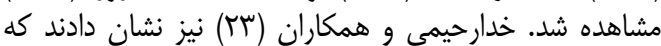

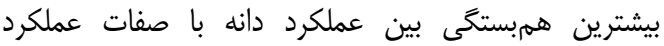

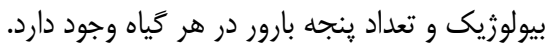

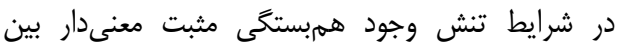

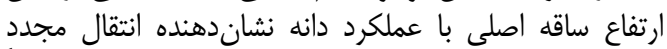

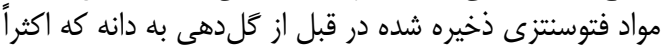

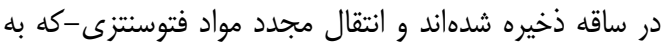

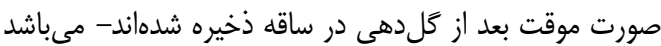

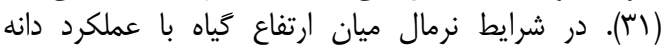

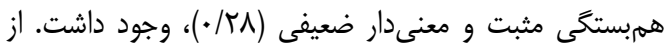


جدول צ- ضرايب همبستخى فنوتيبى ساده بين صفات مختلف در ارقام گندم در شر ايط عدم تنش (آبارى نرمال)

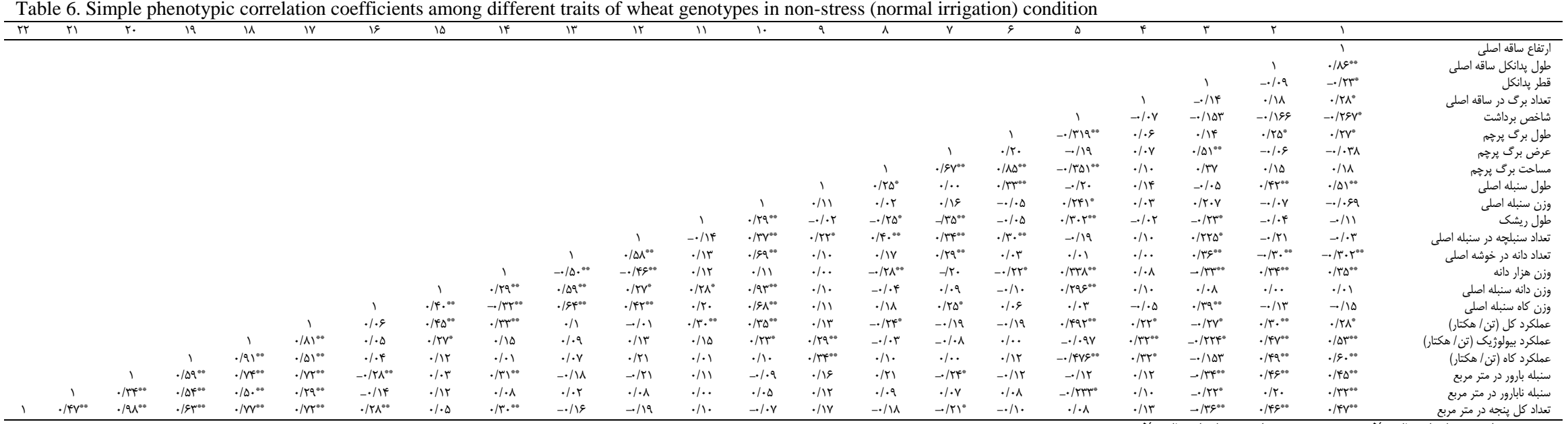

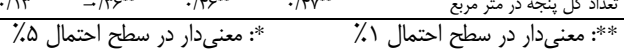

جدول V- ضرايب همبستخى فنوتيبى ساده بين صفات مختلف در ارقام كَندم در شرايط تنش رطوبتى Table 7. Simple phenotypic correlation coefficients among different traits of wheat genotypes under water stress condition

\begin{tabular}{|c|c|c|c|c|c|c|c|c|c|c|c|c|c|c|c|c|c|c|c|c|c|c|}
\hline$\pi$ & $r$ & $r \cdot$ & 19 & 11 & IV & 19 & 10 & 15 & Ir & Ir & 11 & 1. & 9 & 1 & $\mathrm{v}$ & 9 & $\Delta$ & r & $r$ & $r$ & 1 & \\
\hline & & & & & & & & & & & & & & & & & & & & & 1 & ارتثاع ساقه الطيلى \\
\hline & & & & & & & & & & & & & & & & & & & & 1 & $\cdot \mid / 19^{\circ}$ & طول لدانكل ساقه اصلى \\
\hline & & & & & & & & & & & & & & & & & & 1 &.$/$. & $\begin{array}{l}.1 .9 \\
.1 .4\end{array}$ & . & 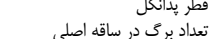 \\
\hline & & & & & & & & & & & & & & & & & 1 &.$- / .4$ & הזו/.- & . &.$/ 1$ & شاخص برداشت \\
\hline & & & & & & & & & & & & & & & & 1 &.$/ 11$ &.$/$. &.$/ 49^{\circ}$ &.$/ 1 r$ & .1 .1 & طول برى بريهم \\
\hline & & & & & & & & & & & & & & & 1 &.$/ / \mathrm{V}$ &.$- / 11$ &.- .1 .1 &.$/ 199 *$ & $-/ 11$ & $-\cdot / \cdot v \mid$ & عرض برك يرحما \\
\hline & & & & & & & & & & & & & & 1 & .194000 & $\cdot \mid N^{600}$ &.$- / 19 \mathrm{~A}$ &. .1 .1 & .1490 & .1 .4 &.$/ T T$ & مساحت برك ي برحئم \\
\hline & & & & & & & & & & & & & 1 & $\cdot \pi V^{*}$ &.$/$. & $\cdot|\Delta|^{s e n}$ & -1.4 & . Mro & .1 .5 &. &.$/ \% 9 *$ & طول سنبله اصلى \\
\hline & & & & & & & & & & & & 1 &.$/ 2 r$ &.$/ 4 \cdot 0$ &.$/ \pi \Lambda^{*}$ & $\cdot \pi I^{*}$ &. $\mid F \% \Lambda^{* *}$ & -.1 .0 &.$/ R 9^{\circ}$ & $\cdot r$. & $\cdot 11$ & وزن سنبله اصلى \\
\hline & & & & & & & & & & & 1 &.$/ 4 \cdot .6$ & $-* /$. &.$- / .+4$ & 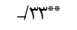 & $\cdot 1 / \Delta$ & $\cdot / \pi r^{\circ}$ & -.1 .4 & $-\cdot / 10$ & $\cdot 11$. & -1.0 & طول ريشك \\
\hline & & & & & & & & & & 1 &.$- / 10$ &.$/ 49^{\circ 0}$ & $\cdot \pi \varphi^{\circ}$ &.$/ F F^{600}$ &.$/ 4 \%$ &.$/ q^{* a+}$ &.$\% r$ &.$/ . v$ &.$/ \kappa^{\circ} \cdots$ & $-\pi / 4 r^{\circ}$ & -1.9 & تعداد سنبلجه در سنبله اصلى \\
\hline & & & & & & & & & 1 &.$/ \Delta F^{*}$ &.$/ 11$ &.$/ Y^{\infty+\infty}$ & .1 .4 &.$/ V^{* 00}$ & . &.$/ \pi \Lambda^{\circ}$ &.$/ 995$ &.$- / . v$ &. &.$- / . v$ & $-/ / 11$ & تعداد دانه درخ خوشه اصلى لى \\
\hline & & & & & & & & 1 &.$- / 4 \cdot a$ & $.0 / 45 *$ & .110 &.$/ 1 \mathrm{~A}$ &. .1 .1 &.$- / 7 \Delta^{\circ}$ &.$- / 19$ &.$/ r$. & . Frro" &.$M$ & $\rightarrow / r \Delta^{\circ}$ & $.1 \% 0^{\circ \infty}$ &.$/ 4400$ & وزن هزار دانه \\
\hline & & & & & & & 1 & . & $.199^{\circ \circ}$ &.$/ \pi 0^{\circ}$ & $.149^{\circ}$ & $. / q \%=0$ & .1 .4 & .119 &.$/ 1 \mathrm{~V}$ &.$/$. & $.|\Delta|\rangle^{* 0}$ &.$- /$. & .114 & $.1 \pi 0^{\circ}$ &.$/ 19 \mathrm{~V}$ & وزن دانه سنبله اصلي \\
\hline & & & & & & 1 &.$/ \mu \Lambda^{\circ}$ & $-\cdot / 19$ & $\cdot|\Delta|^{\circ \infty}$ &.$/ 4 \cdot \cdots$ & $.150^{\circ}$ & $.19 \Lambda^{\circ 0}$ &.$/ K 4^{\circ}$ &.$/ 49^{600}$ & . $/ \mu \Lambda^{* *}$ &.$/ 4 \Delta^{\circ *}$ & $\cdot 11 \cdot 1$ &.$- / . r$ & $.4 \%$ & /.r & $-1 . \%$ & وزن كاه سنبله اصلى \\
\hline & & & & & 1 & $.1 .9 \mathrm{~V}$ & 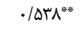 & .14900 & $\cdot / / \mathrm{V}$ &.$/ . r$ & .198 &.$/ 4 \varphi^{600}$ & $\cdot / 1 \Delta$ &.$- / .+$ &.$- / . \mathrm{v}$ & $. .1 . r$ & $. / N+40$ &.$- \% r$ &.$- / 10$ &.$/ 4 V^{50}$ &.$/ F \varphi^{20 *}$ & عملكرد كل (تن/ هكتار) \\
\hline & & & & 1 & $\cdot / 19^{\circ 0}$ &.$/ .4$ &.$/ 4 \varphi^{\infty}$ & . & $\cdot / 1 F$ &.$\%$ &.$/ \cdot v$ & $.49^{\circ}$ &.$/ \pi \varphi^{\circ}$ &.$/ .$. & -.1 .4 &.$/ .$. &.$/ F V \Delta^{\circ *}$ &.$/$. &.$- / 11$ & $.10 \cdot \cdots$ &.$\Delta \Delta^{\circ}$ & عملكرد بيولوزيك (تن/ هكتار) \\
\hline & & & 1 &.$/ 9400$ & $.19 y^{000}$ & .1 .1 &.$/ r \cdot \cdots$ & 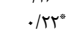 &.$\mu$ &.$/ . r$ &..$/$. &.$/ \mu^{\circ}$ &.$/ T V^{\circ}$ & .1 .4 & .1 .4 & .1 .4 & .1 .4 &.$/ .$. & -.1 .9 & $.140^{\circ 0}$ &.$/ \Delta \gamma^{\circ}$ & عملكرد كاه (تن/هكتار) \\
\hline & & 1 & $.109^{\circ \circ}$ &.$/ 9 \mathrm{Y}^{\circ \circ}$ & $.190^{\circ 00}$ & $\cdot / \mathrm{rr}$ & .1 .0 &.$\kappa \cdots$ &.$/ / Y$ &.$- / \%$ & $. .1 . r$ & .0 .9 &.$/ 11$ & $.1 \varphi^{\circ}$ &.$/ / Y$ &..$/ \pi 0^{\circ}$ &.$/$ / 99** &. .1 .4 &..$/\left.\%\right|^{* 0}$ &. &.$/ 4 \cdots$ & سنبله بارور در متر مربع \\
\hline & 1 &.$/ \mu \cdots$ & $\cdot \pi r^{\circ}$ & .110 & .1 .48 & $-/ \pi r^{\circ}$ & -1.1 & .119 & $-\cdot / 1 \Lambda$ &.$- / 14$ &.$/ 1$ & $-1 / \Delta$ &.$/ 18$ &.$- / T \Lambda^{* *}$ & $-\cdot / 4$ &.$- / \mu \cdot \omega$ &.$- / 19$ & $.4 \mathrm{~V}^{\circ *}$ &.$- / 14$ & $\cdot 1 / 1$ & $. / 4 r=0$ & سنبله نابارور در متر مربع \\
\hline 1 &.$/ \Delta r^{\mathrm{son}}$ &.$/ 9 Y^{\circ 0}$ &.$/ 109^{\circ *}$ & $.199^{900}$ & $.109^{\circ 9}$ & $-\cdot / r^{* * *}$ &.$/ \pi \Lambda$ &.$/ 4 \cdot{ }^{* *}$ & $-* / r$. & $\rightarrow / \pi T^{\circ}$ &.- .1 .1 & -.1 .9 &.$/ 14$ & $-. \mid \mu)^{* 0}(-1)$ &.$- / 11$ & $-\cdot / \mu \cdot{ }^{* * *}$ & . & .1 .99 & $-. / \mu)^{\circ 0}$ &.$/ 4 r^{\circ 0}$ &.$/ 44^{\circ 0}$ & تعداد كل ينجنه در متر مربع \\
\hline
\end{tabular}




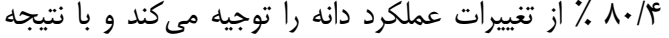

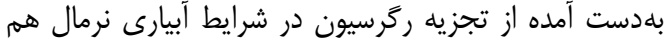

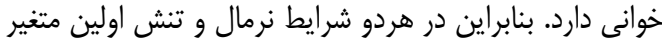

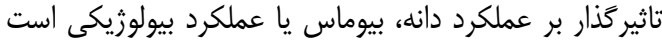

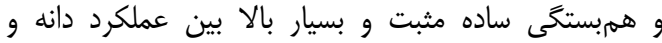

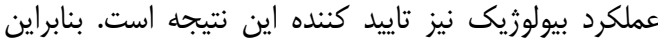

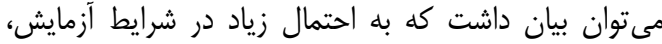

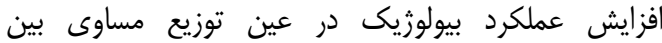

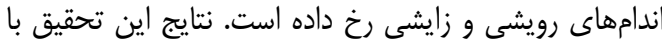

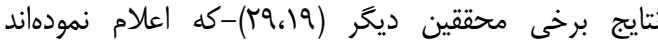

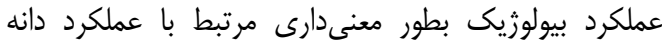

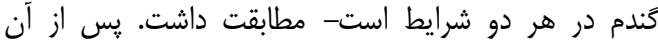

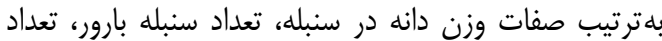

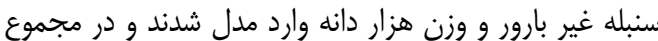

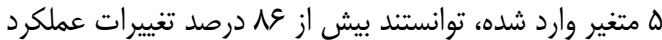

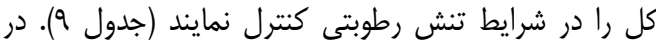

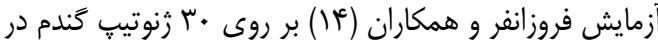

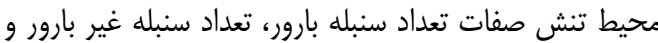

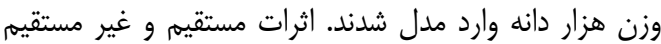

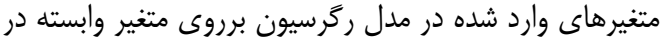
هر دو محيط با استفاده از تجزيه عليت ركائ تفكيك شد (جدول

(1)
در شرايط نرمال، بلترتيب 9 متغير عملكرد بيولوزيك،

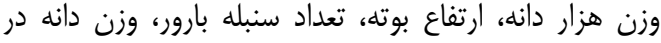

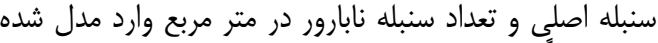

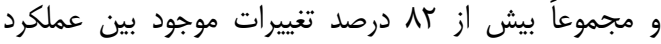

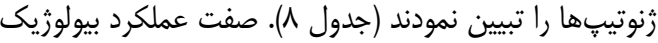

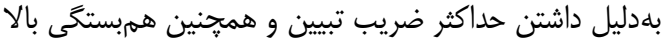

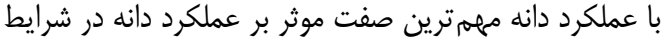

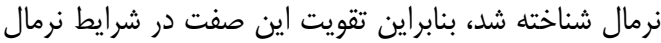

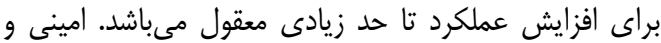

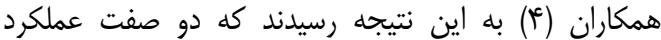

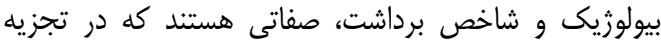

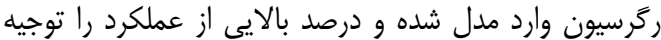

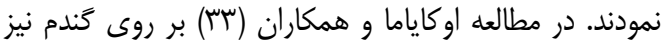

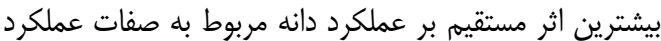

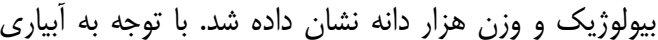

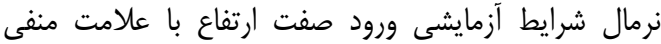

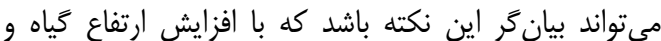

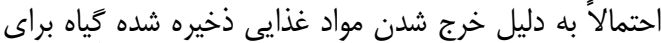

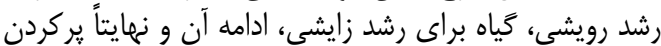

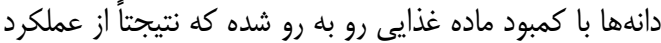

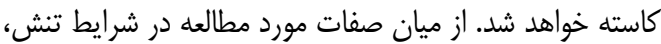
عملكرد بيولوزيك نخستين متغير وارد شده به مدل مدل بود كه

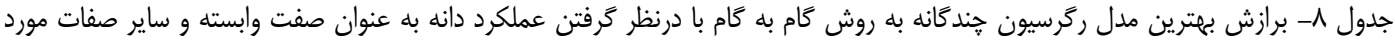

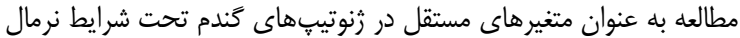

Table 8. Fitness of the best multiple regression model using stepwise method considering grain yield as dependent and other traits as independent tratis of wheat genotypes in normal condition.

\begin{tabular}{|c|c|c|c|c|c|c|c|c|c|c|c|c|}
\hline \multicolumn{2}{|c|}{ كام ششم } & \multicolumn{2}{|c|}{ كَام ينجهم } & \multicolumn{2}{|c|}{ كام جهارم } & \multicolumn{2}{|c|}{ كَام سوم } & \multicolumn{2}{|c|}{ كام دوم } & \multicolumn{2}{|c|}{ كام اول } & \\
\hline خطا & ركرسيون & خطا & ركرسيون & خطا & ركرسيون & خطا & ركرسيون & خطا & ركرسيون & خطا & ركرسيون & \\
\hline$V \Delta$ & 9 & ve & $\Delta$ & W & $r$ & $\mathrm{v \Lambda}$ & $r$ & va & $r$ & $\Lambda$. & 1 & درجه آزادى \\
\hline \multicolumn{2}{|c|}{ سنبله غيربارور در } & \multicolumn{2}{|c|}{ وزن دانه در سنبله } & \multicolumn{2}{|c|}{ سنبله بارور در متر } & \multicolumn{2}{|c|}{ ارتفاع بوته } & \multicolumn{2}{|c|}{ وزن هزار دانه } & \multicolumn{2}{|c|}{ عملكرد بيولوزيك } & صفت وارد شده \\
\hline ./TYq & $1 \mathrm{~N} / \mathrm{Vq}$ &.$/$ HYF & TY/TD & $\cdot / r V q$ & $r V / \cdot V$ & $\cdot / 4 \cdot 1$ & $r \Delta / 19$ & . IQTA & $F V / \Lambda S$ &.$/ 094$ & 19/9r & ميانكين مربعات \\
\hline & 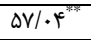 & & 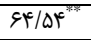 & & $V \backslash / \varphi^{2}$ & & $\Lambda \& / . \sigma^{\prime \prime \prime}$ & & $9 \cdot / \Delta f^{m+1}$ & & $|Q| / T V$ & Fقدار F مق F \\
\hline & . AT & & $\cdot 14.9$ & & - IVAV & & . IVFA & &.$/ 994$ & & - IgDF & ضريب تبيين \\
\hline & . & & ./RANT & &.$/ \cdots v$ & & $\cdot / \cdot \cdot \wedge$ & & . & & 1.1 .49 & خطاى استاندارد \\
\hline & $-.1 . \cdot 99$ & & ./VYVq & & $. / \cdot+\mathrm{rN}$ & &.$- / .4 Q I$ & & $\cdot / \cdot$ TNS & & $\cdot / T T \Delta$ & ضرايب ركرسيون \\
\hline & $\cdot / N{ }^{M} \Lambda$ & &.$/ \& \vee \Delta$ & &.$/$ TOT & & $\cdot / \Delta V T$ & &.$/ 94 \mathrm{~V}$ & &.$/ T \Delta A$ & tolerance \\
\hline & $1 / \pi \Delta$ & & $1 / 4 \wedge$ & & $r / \Lambda \mu$ & & $1 / N F^{c}$ & & $1 / \Delta F$ & & $r / A V$ & VIF \\
\hline
\end{tabular}




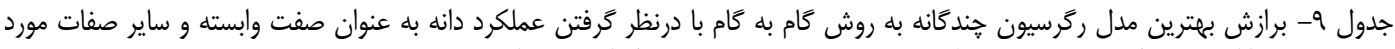

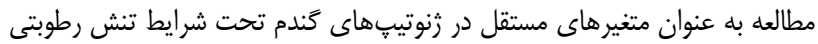

Table 9. Fitness of the best multiple regression model using stepwise method considering grain yield as dependent and other traits as independent tratis of wheat genotypes in water stress condition

\begin{tabular}{|c|c|c|c|c|c|c|c|c|c|c|}
\hline \multicolumn{2}{|c|}{ كام ينجمم } & \multicolumn{2}{|c|}{ كام حهارم } & \multicolumn{2}{|c|}{ كام سوم } & \multicolumn{2}{|c|}{ كام دوم } & \multicolumn{2}{|c|}{ كام اول } & \\
\hline خطا & ركرسيون & خطا & ركرّيون & خطا & ر ركرسيون & خطا & ر رَرسيون & خطا & ركر سيون & \\
\hline Ve & $Q$ & w & r & VA & $r$ & vq & $r$ & $\Lambda$. & 1 & درجه آزادى \\
\hline \multicolumn{2}{|c|}{ وزن هزار دانه } & \multicolumn{2}{|c|}{ سنبله غيربارور در متر مربع } & \multicolumn{2}{|c|}{ سنبله بارور در متر } & \multicolumn{2}{|c|}{ وزن دانه در سنبله } & \multicolumn{2}{|c|}{ عملكرد بيولوزيك } & صفت وارد شده \\
\hline \multirow[t]{7}{*}{. } & $\mid r / V r$ &.$/$ lfy & $\mid \mathrm{V} / \cdot \cdot$ &.$/ 1 Q V$ & $r T / r q$ &.$/ M n$ & $r r / \Lambda$. &.$/ 194$ & $94 / 99$ & ميانكين مربعات \\
\hline & $99 / \Delta \Delta^{+* *}$ & & $11 V / \Delta F^{* *}$ & & 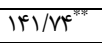 & & $191 / 9 \Lambda^{272}$ & & $r T q / \Lambda e^{* * *}$ & Fقدار F مقار \\
\hline & $\cdot / \Lambda \& V$ & & $\cdot / \wedge \Delta q$ & & $\cdot / A f \Delta$ & & $\cdot /$ /Arq & & $\cdot / \Lambda \cdot F^{2}$ & ضريب تبيين تجمعى \\
\hline &.$/ . .9 \mathrm{~V}$ & &.$/ \cdot \cdot r$ & & $\cdot / \cdots \Delta$ & & $\cdot / r \cdot \Delta f$ & & 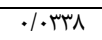 & خطاى استاندارد \\
\hline &.$/ . K I T$ & &.$- / . \cdot \mu \Lambda$ & & $\cdot / \cdot \cdot 10$ & & $\cdot / R T I K$ & & $\cdot / \mu r+1$ & ضرايب ركر سيون \\
\hline & $\cdot / V^{\mu} S$ & & ./194 & & . & & $. / 8 \mid 1$ & & $\cdot / r q q$ & tolerance \\
\hline & $1 / \pi \Delta$ & & $1 / T$ & & T/QT & & $1 / 94$ & & $r / V \cdot$ & VIF \\
\hline & & & & & عرض از مـ & & حتمال ه. & ار در ט & o:* & ىدار در سطح احتمال ٪ \\
\hline
\end{tabular}

جدول • ا- اثرات مستقيم (ضرايب عليت) و غيرمستقيم صفات مختلف برعملكرد دانه در شرايط بدون تنش (آبيارى نرمال) با استفاده از ماتريس همبستخى فنوتيبى بين صفات (ضراتي عليت

Table 10. Direct (path coefficients) and indirect effects of different traits on grain yield in non-stress (normal irrigation) condition using phenotypic correlation matrics among traits.

\begin{tabular}{|c|c|c|c|c|c|c|c|}
\hline مقدار كل (همبستخى) & سنبله غيربارور (متر مربح) & وزن دانه در سنبله & (متبله بارور & $\begin{array}{l}\text { ارتفاع بوته } \\
\text { بوت }\end{array}$ & وزن هزار دانه & عملكرد بيولوزيك & \\
\hline$\left.\cdot|\mathrm{A}| \cdot\right|^{* * a}$ & $-.1 .9 . V$ & .1 .499 &.$/ \% 199$ &.$- / 1489$ & .1. Tq4 &.$/$.VIY & 1 \\
\hline . &.$- / .1 \cdot r$ & $.1 .+4 \% q$ & .1 .94$. & -.1 .919 & . /Mr. &.$/ 1 \backslash Q S$ & $r$ \\
\hline . / $Y 99^{*}$ & $-. .1 . r q \vee$ & |... & . /Trबq & $-\cdot /$ TYYS & $\overline{.1 .9 \Delta V V}$ & . rAVA & r \\
\hline$\cdot /$ T ${ }^{* * * *}$ &.$-|\cdot+| 9$ & $\cdot / \cdot \lambda$. & ./rq४. & $\overline{-. / I T E V}$ & $.1 . \Delta S V$ & | ATrS & f \\
\hline.$/ F T \Delta F^{* \theta \Delta}$ & זه|.|-. &.$/ 1 V \cdot 1$ & $\overline{.1 .141}$ & $-.1 .+19$ & . & $\cdot / r \cdot W r$ & $\Delta$ \\
\hline.$/ 491 \Lambda^{* * 8}$ & $-\cdot / \pi \cdot \Delta$ & $. / \cdot Y \mid q$ & $\cdot / \cdot r \Delta$ & -.1 .191 &.$/ \cdot 1 \Delta \Delta$ & איאפץ/. & 9 \\
\hline
\end{tabular}

جدول || - اثرات مستقيم (ضرايب عليت) و غيرمستقيم صفات مختلف برعملكرد دانه در شرايط تنش رطوبتى با استفاده از ماتريس همبستخى

Table 11. Direct (path coefficients) and indirect effects of different traits on grain yield in stress condition using phenotypic correlation matrics among traits.

\begin{tabular}{|c|c|c|c|c|c|c|}
\hline مقدار كل (همبستگىى) & وزن هزار دانه & سنبله غير بارور & سنبله بارور & وزن دانه در سنبله & عملكرد بيولوزيك & \\
\hline$\cdot / \Lambda 9 V{ }^{\prime * *}$ & 更. &.$- / .199$ & .//r"q & $\cdot / \cdot 119$ & . & 1 \\
\hline - $/ \Delta T V Q^{* * *}$ & سזץ./. & Trו. &.$/ .19$ & $\cdot / r \cdot \Delta r$ & $\overline{. / \Upsilon 99 \wedge}$ & r \\
\hline . $|90| T^{m * * *}$ & TKF & קq & . MAYE &.$/ .9$. & $\cdot / F \Delta V I$ & r \\
\hline$\cdot / \cdot r \Delta \gamma^{n s}$ & .1 .191 & אוM/R & $\cdot \cdot \cdot \Delta \Delta \mid$ &.$- / \cdot \ln$ & שr & f \\
\hline . & $\cdot 11 \cdot 19$ & $\overline{-* / .4 \mid .}$ &.$\cdot v{ }^{\prime} \Delta$ & .1 .9 .9 & אוצוT/. & $\Delta$ \\
\hline
\end{tabular}

عملكرد دانه مربوط به صفت ارتفاع بوته مىباشد به عبار إنهارت

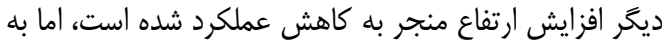

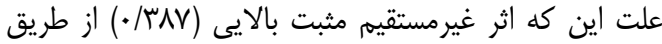

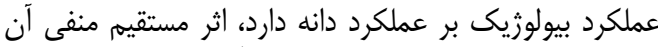

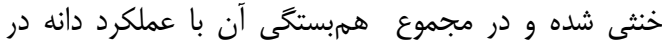

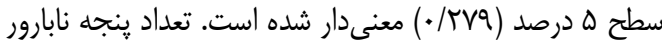

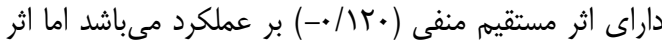
غيرمستقيم مثبت بالاى آن از طريق آنقيم عملكرد بيولوزيك اثر

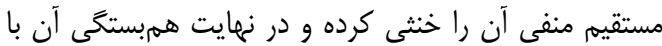

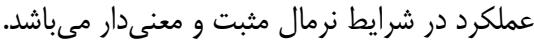

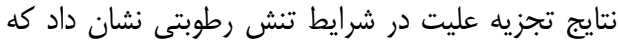

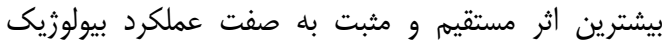

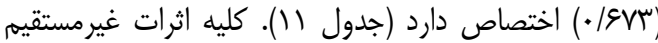

نتايج نشان داد در شرايط نرمال (جدول • (1) بالاترين اثر

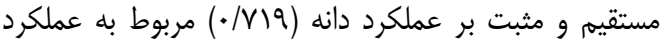

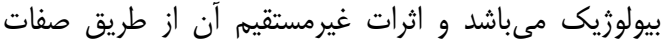

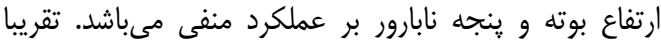

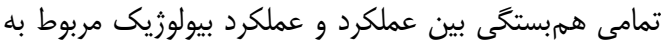
اثر مستقيم اين صفت مى باشد. در مطالعه كَّليرور و همكاران (1)

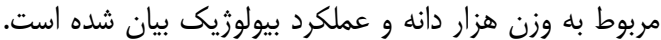

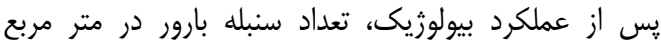

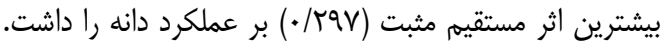

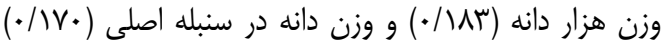

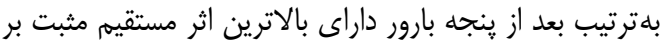

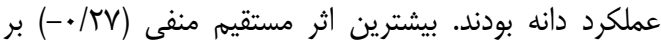




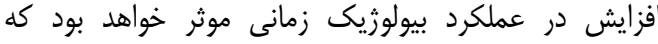

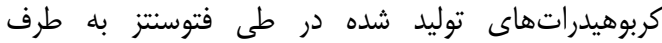

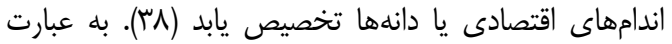

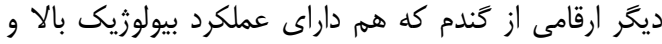

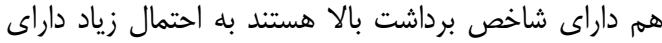

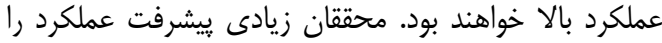

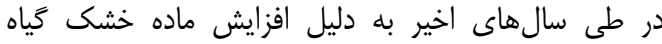

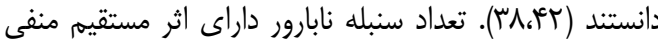

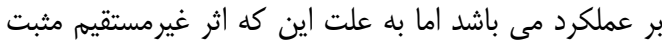

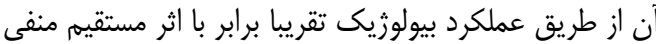

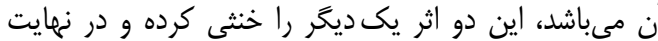

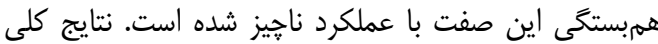

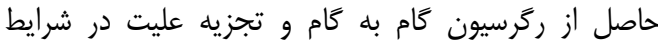

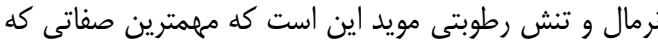

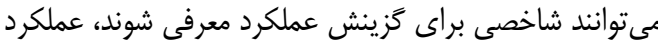

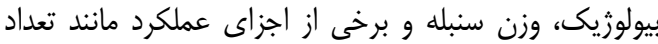

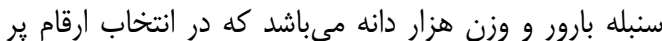

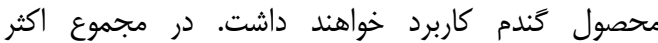

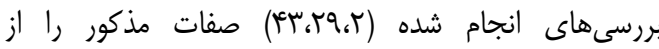

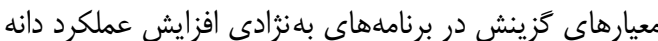

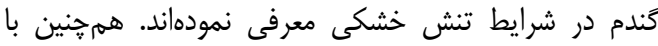

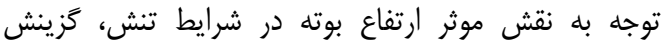

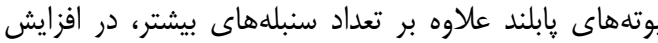

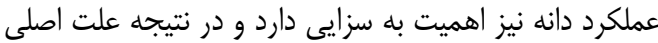

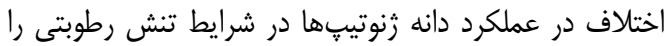
مىتوان به تفاوت در اين صفات نسبت داد دون دان

$$
\text { تشكر و قدردانى }
$$

بدين وسيله از جناب آقاى مهرئدس عليرضا كرزى و و دكتر

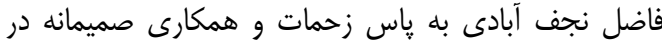
اجراى اين طرح قدردانى مىشود.

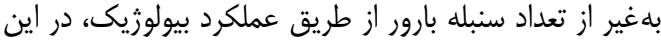

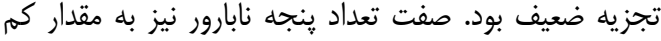

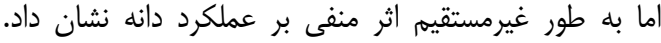

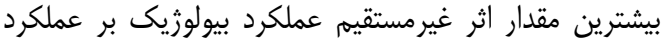

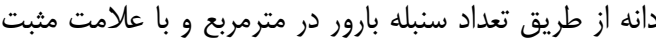

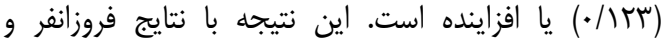

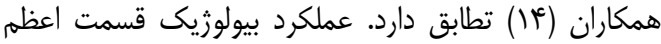

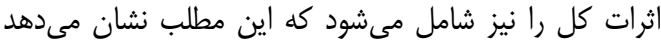

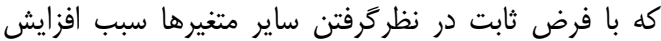

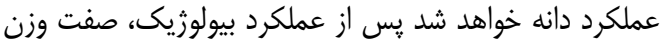

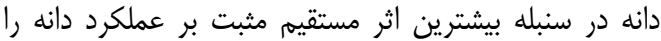

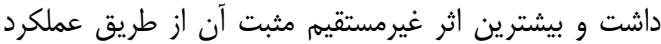

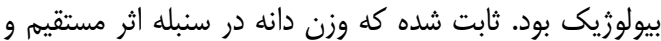

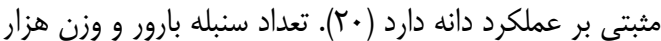

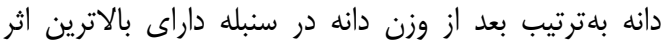

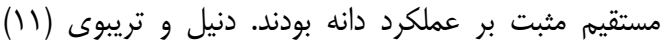

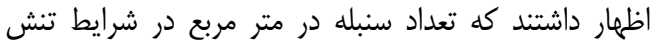

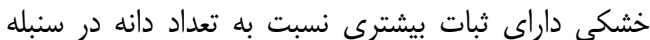

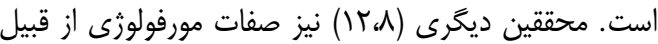

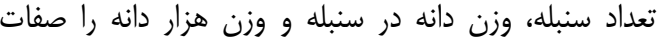

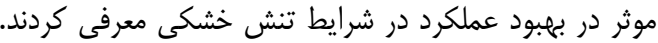

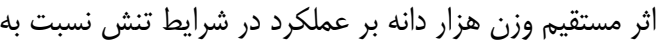

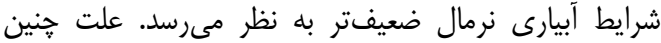

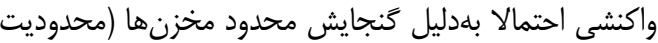

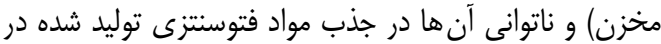

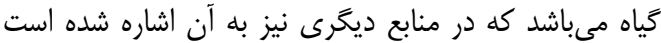

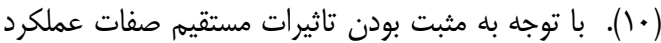

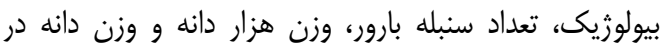

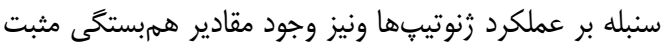

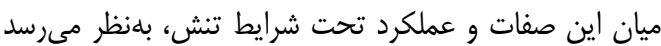

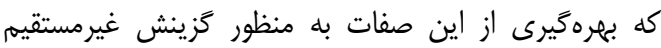

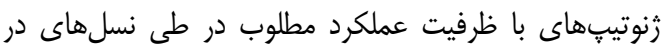

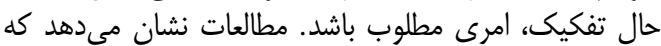



genotype collection of Iran. Seed and Plant Improvement Journal, 27: 581-599 (In Persian).

2. Ahmadizadeh, M., M. Valizadeh, H. Shahbazi, M. Zaefizadeh and M. Habibpor. 2011. Morphological diversity and interrelationships traits in durum wheat landraces under normal irrigation and drought stress conditions. Advances in Environmental Biology, 5: 1934-1940.

3. Alamerew, S., S. Chebotar, X. Huang, M. Roder and A. Borner. 2004. Genetic diversity in Ethiopian hexaploid and tetraploid wheat germplasm assessed by microsatellite markers. Genetic Resources and Crop Evolution, 51: 559-567.

4. Amini, A., M. Esmailzade-Moghadam and M. Vahabzadeh. 2005. Genetic diversity based on agronomic performance among Iranian wheat Landraces under moistur stress. Proc. The 7th international wheat conference, Nov. 27- Dec 2, 2005. Mardel Plata-Argentina.

5. Babu, R.C., J. Zhang, A. Blum, T.H.D. Ho, R. Wu and H.T. Nguyen. 2004. HVA1, a LEA gene from barley confers dehydration tolerance in transgenic rice (Oryza sativa L.) via cell membrane protection. Plant Science, 166: 855-862.

6. Beltrano, J. and M.G. Ronco. 2008. Improved tolerance of wheat plants (Triticum aestivum L.) to drought stress and rewatering by the arbuscular mycorrhizal fungus Glomus claroideum: effect on growth and cell membrane stability. Brazilian Journal of Plant Physiology, 20: 29-37.

7. Blum, A. 2005. Mitigation of drought stress by crop management. Available online at:http://www.plant stress.com.

8. Blum, A. 2005. Mitigation of drought stress by crop management. available at: www. Plant Stress.com..article.drought_m.drought_m.htm.mitigation_by_management.

9. Bogale, A., K. Tesfaye and T. Geleto. 2011. Morphological and physiological attributes associated to drought tolerance of Ethiopian durum wheat genotypes under water deficit. Journal Of Biodiversity and Environmental Sciences, 1: 22-36.

10. Borras, L., G.A. Slafer and M.G. Otegui. 2004. Seed dry weight response to source-sink manipulation in wheat, maize and soyabean. A quantitative reapprasial. Field Crops Research, 86: 131-146.

11. Daniel, C. and E. Triboi. 2002. Changes in wheat protein aggregation during grain development: Effects of temperatures and water stress. European Journal of Agronomy, 16: 1-12.

12. Eskandari, H. and K. Kazemi. 2010. Response of different bread wheat (Triticum aestivum L.) genotypes to post-anthesis water deficit. Notulae Scientia Biologicae, 2: 49-52.

13. Fischer, R.A. 2008. The importance of grain or kernel number in wheat: A replay to Sinclair and jamieson. Field Crop Research, 105: 15-21.

14. Foroozanar, M., M. Bihamta, A. Peyghambary and H. Zali. 2012. Evaluation of bread wheat genotypes under normal and water stress conditions for agronomic traits. Journal of Sustainable Agriculture and Production Science, 21: 33-46 (In Persian).

15. Foulkes, M.J. 2002. The ability of wheat cultivars to withstand drought in UK conditions: formation of grain yield. Journal Agriculture of Science, 38: 153-169.

16. Garavandi, M., E. Farshadfar and D. Kahrizi. 2010. Assessm ent of drought stress in advanced wheat genotypes under normal and controlled conditions. Seedling and Seed, 26: 233-252 (In Persian).

17. Golabadi, M., A. Arzani and S.M.M. Maibody. 2005. Evaluation of variation among durum wheat F3 families for grain yield and its components under normal and water-stress field conditions Czech. Journal of Genetic and Plant Breeding, 41: 263-267.

18. Golparvar, A.R., M.R. Ghanadha, A.A. Zali, A. Ahmadi, E.M. Heravan and A. Ghasemipirbalooti. 2005. Factor analysis of morphological and morpho-physiologocal traits in bread wheat (Triticum aestivum L.) genotypes under drought and non-drought stress conditions. Pajouhesh and Sazandegi, 72: 52-59 (In Persian).

19. Guendouz, A., S. Gussoum, K. Maamari and M. Hafsi. 2012. Effect of supplementary on grain yield, yield components and some morphological traits of Drum Wheat (Triticum Durum Desf.) cultivar. Advances in Environmental Biology, 6: 564-572.

20. Guoth, A., I. Tari, A. Galle, J. Csiszar, A. Pecsvaradi, L. Cseuz and L. Erdei. 2009 . Comparison of the drought stress responses of tolerant and sensitive wheat cultivars during grain filling: Changes in flag leaf photosynthetic activity, ABA levels and grain yield Journal of Plant Growth Regulation, 28: $167-176$.

21. Heidari sharifabad, H. 2008. Drought mitigation strategies for the agriculture sector. The $10^{\text {th }}$ Iranian congress of Crop Science, 18-20 Aug. 2008, SPII, Karaj, Iran (In Persian).

22. Khan, A.S., S. Ul-Allah and S. Sadique. 2010. Genetic variability and correlation among seedling traits of Wheat (Triticum aestivum) under water stress. International Journal of Agriculture and Biology, 2: 247-250.

23. Khayatnezhad, M., M. Zaeifizadeh, R. Gholamin and Sh. Jamaati-e-somarin. 2010. Study of genetic diversity and path analysis for yield in durum wheat genotypes under water and dry conditions. World Applied Sciences Journal, 9: 655-665.

24. KhodaRahmi, M., A. Amini and M.R. Bihamta. 2006. study of traits correlation and path analysis grain yield triticale. Iranian Journal of Agricultural Science, 1-37: 77-83 (In Persian).

25. Kiliç, H. and T. Yağbasanlar. 2010. The Effect of Drought Stress on Grain Yield, Yield Components and some Quality Traits of Durum Wheat (Triticum turgidum ssp. durum) Cultivars. Notulae Botanicae Horti Agrobotanici Cluj-Napoca, 38: 164-170.

26. Kotal, B.D., A. Das and B.K. Choudhury. 2010 . Genetic variability and association of characters in wheat (Triticum aestivum L). Asian Journal of Crop Science, 2: 155-160. 
27. Moayedi, A.A., A.N. Boyce and S.S. Barakbah. 2010. The performance of durum and bread wheat genotypes associated with yield and yield component under different water deficit conditions. Australian Journal of Basic and Applied Sciences, 4: 106-113.

28. Mohammadi, V., M.R. Qannadha, A.A. Zali and B. Yazdi-Samadi. 2010. Effect of Post Anthesis Hear Stress on Head Traits of Wheat. International Journal of Agriculture and Biology, 1: 42-44.

29. Mohammadi, A., E. Majidi Heravan, M.R. Bihamta and H. Heidari Sharifabad. 2007 . Evaluation of drought stress on agro-morphological characteristic in some wheat cultivars. Pajouhesh and Sazandegi, 73: 184-192 (In Persian).

30. Moral, G.L.F., Y. Rharrabti, D. Villegas and C. Royo. 2003. Evaluation of grain yield and its components in durum wheat under Mediterranean conditions: An ontogenic approach. Agronomy Journal, 95: 266-274.

31. Nofouzi, F., V. Rashidi and A.R. Tarinejad. 2008. Path Analysis of Grain Yield with Its Components in Durum Wheat under Drought Stress. International Meeting on Soil Fertility Land Management and Agroclimatology. Turkey, pp: 681-686.

32. Nouri-Ganbalani, A., G. Nouri-Ganbalani and D. Hassanpanah. 2009. Effects of drought stress condition on the yield and yield components of advanced wheat genotypes in Ardabil. Iranian Journal of Food Agriculture and Environment, 7: 228-234 (In Persian).

33. Okuyama, L.A., L.C. Fedrizzi and J.F. Barbosa. 2004. Correlation and path analysis of yield and its components and plant traits in wheat. Ciencia Rural, 34: 1701-1708.

34. Passioura, J.B. 2007. Increasing crop productivity when water is scarce-from breeding to field management. Agricultural Water Management, 80: 176-196.

35. Rawson, H.M., R.A. Richards and R. Munns. 1988. An examination of selection criteria for salttolerance in wheat, barley and triticale genotypes. Australian Journal of Agricultural Research, 39: 759-772.

36. Rebetzke, G.J., R.A. Richards, A.G. Condon and G.D. Farquhar. 2006. Inheritance of carbon isotope discrimination in bread wheat (Triticum aestivum L.). Euphytica, 150: 97-106.

37. Reddy, A.R., K.V. Chaitanya and M.V. Vivekanadan. 2004. Drought-induced responses of photosynthesis and antioxidant metabolism in higher Plants. Plant Physiology, 161: 1189-1202.

38. Reynolds, M., M.J. Foulkes, G.A. Slafer, P. Berry, M.A.J. Parry, J.W. Snape and W.J. Angus. 2009. Raising yield potential in wheat. Journal of Experimental Botany, 60: 1899-1918.

39. Royo, C., N. Aparicio, R. Blanco and D. Villegas. 2004. Leaf and green area development of durum wheat genotypes grown under Mediterranean conditions. European Journal of Agronomy, 20: 419430 .

40. Saeidi, M., F. Moradi, A. Ahmadi, R. Spehri, G. Najafian and A. Shabani. 2010. The effects of terminal water stress on physiological characteristics and sink-source relations in two bread wheat (Triticum aestivum L.) cultivars. Iranian Journal of Crop Science, 12: 392-408 (In Persian).

41. Shamsi, K. 2010. The effects of drought stress on yield, relative water content, proline, soluble carbohydrates and chlorophyll of bread wheat cultivars. Journal of Animal and Plant Sciences, 8: 1051-1060.

42. Shearman, V.J., R. Sylvester-Bradley, R.K. Scott and M.J. Foulkes. 2005. Physiological processes associated with wheat yield progress in the UK. Crop Science, 45: 175-185.

43. Shepherd, A., S.M.C.M. Ginn and G.C.L. Wyseure. 2002. Simulation of the effect of water shortage on the yields of winter wheat in North-East England. Ecological Modeling, 147: 41-52.

44. Shutz, M. and A. Fangmeier. 2001. Growth and yield responses of spring wheat (Trithcum aestivum L. cv.Minaret) to elevated co2 and water limitation. Environmental Pollution, 119: 187-194.

45. Singh, B.N., S.R. Vishwakarma and V.K. Singh. 2010. Character association and path analysis in elite lines of wheat (Triticum aestivum L.). Plant Archives, 10: 845-847.

46. Tahmasebi, S., M. Khodambashi and A. Rezai. 2007. Estimation of genetic parameters for grain yield and related traits in wheat using diallel analysis under optimum and moisture stress conditions, Journal of Agricultural Sciences and Natural Resources, 1: 229-240 (In Persian).

47. Vij, S. and A.K. Tyagi. 2007. Emerging trends in the functional genomics of the abiotic stress response in crop plants. Journal of Plant Biotechnology, 5: 361-380.

48. Zhe, Y., J.G. Lauer, R. Borges and N. de Leon. 2010. Effects of Genotypex Environment Interaction on Agronomic Traits in Soybean. Crop Science, 50: 696-702. 


\title{
Evaluation of Bread Wheat Genotypes under Normal and Post-anthesis Drought Stress Conditions for Agronomic Traits
}

\author{
Mandana Mohseni ${ }^{1}$, Seyyed Mohammad Mahdi Mortazavian ${ }^{2}$, Hossein Ali Ramshini ${ }^{3}$ and \\ Behrooz Foghi ${ }^{4}$ \\ 1, 3 and 4- Graduated M.Sc., Assistant Profeesor and Instructore, College of Aburaihan-University of Tehran \\ 2- Associate Professor, College of Aburaihan-University of Tehran \\ (Corresponding author: mortazavian@ut.ac.ir) \\ Received: June 19, $2014 \quad$ Accepted: July 26, 2014
}

\begin{abstract}
In order to study the effect of drought stress and determination of effective traits on grain yield of wheat in normal and terminal drought stress conditions, 82 wheat genotypes were studied in two separate experiments using randomized complete block design with three replications during cropping season 2011-2012. Drought stress was applied in heading stage by limited irrigation. Set of 22 traits including yield and yield components were measured. Results of analysis of variance showed that in both conditions there are significant different between all genotypes for all traits. Drought stress decreased all traits except number of leaves on the main stem and the number of tillers. The highest genetic diversity in two conditions were for awn length, grain yield and number of fertile tiller. Pearson simple correlation, stepwise regression analysis and path analysis in both conditions on all traits took over. In stress condition amount of correlation between grain yield with biological yield, straw yield, harvest index, Fertile spikes per square meter and total number of tillers per square meter equal to or greater than $50 \%$ was achieved. In both environments, the highest correlation was found between biological yield and grain yield. The results indicate the importance of peduncle length in becoming greater amount of grain yield under drought condition. Based on stepwise regression analysis in normal irrigation 6 variables and in stress conditions 5 variables entered the model and in total more than $80 \%$ of the changes were justified. To determine the direct and indirect effects of traits on yield, path coefficient analysis was done, the most direct effect on grain yield in both conditions belonged to biological yield. Based on this study the most important traits that are introduced as an indicator for selection high yielding varieties of wheat in both conditions, are biological yield, spike weight, number of fertile spike and 1000 kernel weight. Also the effective role of height plant in stress condition, selection of higher plants in addition to more spikes in increasing grain yield be important.
\end{abstract}

Keywords: Correlation, Limited irrigation, Path analysis, Stepwise regression, Yield component 